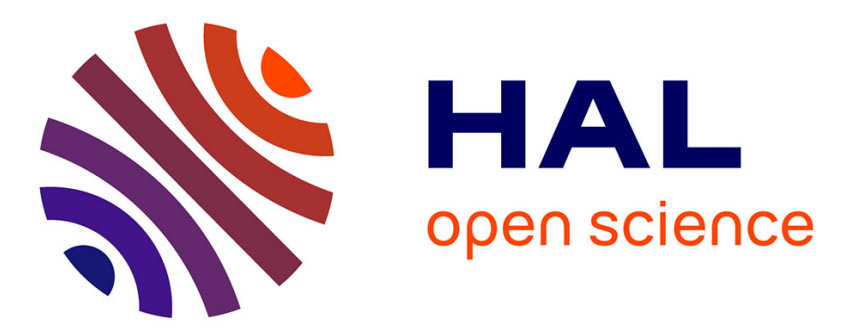

\title{
A posteriori analysis of a finite element discretization of a Naghdi shell model
}

Hervé Le Dret, Christine Bernardi, Frédéric Hecht, Adel Blouza

\section{To cite this version:}

Hervé Le Dret, Christine Bernardi, Frédéric Hecht, Adel Blouza. A posteriori analysis of a finite element discretization of a Naghdi shell model. IMA Journal of Numerical Analysis, 2012, 33 (1), pp.190-211. 10.1093/imanum/drs009 . hal-01418420

\section{HAL Id: hal-01418420 \\ https://hal.sorbonne-universite.fr/hal-01418420}

Submitted on 16 Dec 2016

HAL is a multi-disciplinary open access archive for the deposit and dissemination of scientific research documents, whether they are published or not. The documents may come from teaching and research institutions in France or abroad, or from public or private research centers.
L'archive ouverte pluridisciplinaire HAL, est destinée au dépôt et à la diffusion de documents scientifiques de niveau recherche, publiés ou non, émanant des établissements d'enseignement et de recherche français ou étrangers, des laboratoires publics ou privés. 


\title{
A posteriori analysis of finite element discretizations of a Naghdi shell model
}

\author{
by Christine Bernardi ${ }^{1}$, Adel Blouza ${ }^{2}$, Frédéric Hecht ${ }^{1}$, and Hervé Le Dret ${ }^{1}$
}

\begin{abstract}
We consider two finite element discretizations of the Naghdi equations which model a thin three-dimensional shell. Both of them are derived from a mixed formulation of these equations, and a penalty term is added in the second one. The a posteriori analysis of the discrete problems leads to the construction of error indicators which satisfy optimal estimates. We describe a mesh adaptivity strategy relying on these indicators and we present some numerical experiments that confirm its efficiency.
\end{abstract}

Résumé: Nous considérons deux discrétisations par éléments finis des équations de Naghdi qui modélisent une coque tridimensionnelle de faible épaisseur. Les deux problèmes discrets sont construits à partir d'une formulation mixte de ces équations, avec un terme de pénalisation supplémentaire dans le second. L'analyse a posteriori de ces problèmes mène à la construction d'indicateurs d'erreur qui satisfont des estimations optimales. Nous proposons une stratégie d'adaptation de maillage basée sur ces indicateurs et présentons quelques expériences numériques qui confirment son efficacité.

1 Université Pierre et Marie Curie-Paris6, UMR 7598 LJLL, Paris, F-75005 France; CNRS, UMR 7598 LJLL, Paris, F-75005 France.

2 Laboratoire de Mathématiques Raphaël Salem (UMR 6085 CNRS), Université de Rouen, avenue de l'Université, B.P. 12, F-76801 Saint-Étienne-du-Rouvray, France.

e-mail addresses: bernardi@ann.jussieu.fr, Adel.Blouza@univ-rouen.fr, hecht@ann.jussieu.fr, ledret@ann.jussieu.fr 


\section{Introduction.}

Naghdi's equations model linearly elastic shells. In their classical formulation, they constitute a system of linear second-order partial differential equations which is elliptic when the shell is clamped on part of its boundary: The unknowns are the covariant components of the displacement of the shell and of its rotation. A further constraint is enforced on the rotation which must be tangent to the shell; this constraint is easily expressed in covariant components. However, such a formulation does not accomodate curvature discontinuities on the shell midsurface.

The formulation of Naghdi's model which is used here was introduced by Blouza [7] and Blouza and Le Dret [10]. It relies on the idea of using a local basis-free formulation in which the unknowns are described in Cartesian coordinates instead of covariant or contravariant components as is usually done in shell theory, see for example [2]. Such a formulation is able to handle shells with a $W^{2, \infty}$-midsurface. In particular, midsurface curvature discontinuities are allowed. Moreover, in view of the discretization and as first proposed in [8], a Lagrange multiplier can be introduced to handle the tangency requirement on the rotation. This leads to a well-posed mixed variational problem.

The literature on finite element approximation of two-dimensional shell models is large. Let us mention a few approaches. Concerning conforming methods, the Ganev and Argyris triangles provide interpolation by polynomials of degree 4 and 5, with high order convergence in $c h^{4}$ when the solution is smooth enough. These elements are used for example to study the linear Koiter model for $\mathscr{C}^{3}$-shells in the classical covariant formulation, see [1, Part. II, Chap. 1]. Such methods are also applied to approximate geometrically exact shell models in [11]. The Argyris elements are used in [18] for numerical analysis of Koiter's model with little regularity in the Cartesian formulation proposed in [9]. We also mention the 3-dimensional shell element approach, see [13]. Still in the context of shells with little regularity, i.e., when the midsurface is of $W^{2, \infty}$-regularity, a non conforming DKT (discrete Kirchhoff triangle) element is used in [21] to approximate Koiter model. Other works [19][20] concern the finite element discretization of shell problems with domain decomposition.

In this work, we are interested in two other finite element discretizations relying on the mixed formulation and already studied in [8]. In the second one, a penalty term is added to the mixed formulation as standard for saddle-point problems, see [16, Chap. I, $\S 4.3]$, which leads to an efficient algorithm for solving the resulting linear system. The convergence of both discretizations is proved in [8], where a complete a priori analysis is performed and numerical tests are presented.

A posteriori analysis is now an important tool for improving the efficiency of the discretization and, up to our knowledge, has not yet been performed for the discretization of shell models (we refer to [12] for a first work in this direction concerning a plate model). Its first aim is mesh adaptivity. Indeed, a much smaller number of degrees of freedom is needed to obtain a given accuracy when the final mesh is adapted to the solution, and the construction of such a mesh relies on error indicators which only depend on the discrete solution, hence can be computed in an explicit and most often non expensive way. A posteriori estimates prove that these indicators provide a good representation of the local 
error, see [22] for a detailed presentation of all this. So we perform the a posteriori analysis of the first discretization and prove upper and lower bounds for the error as a function of residual type indicators.

In the case of penalty methods such as described in [16, Chap. I, §4.3] for instance, it has been proved in [5] that a posteriori analysis also provides an evaluation of the error issued from the addition of a penalization term (see [4] for another application). This requires two kinds of error indicators: One indicator is linked to the penalization and a family of "local" indicators to the finite element discretization. The main idea of this approach is to uncouple as much as possible the evaluation of the errors stemming from the penalization and from the finite element discretization and to choose the penalty parameter in order that the two errors are of the same order. In this case also, we prove optimal estimates for the error.

In a final step, we describe the strategy which is used in both cases for adaptivity. Numerical experiments are in good agreement with the analysis. They justify our choices of discretization and lead to a comparison of the two methods that we propose.

An outline of the paper is as follows.

- In Section 2, we recall the geometry of the midsurface and Naghdi's equations. Next, we write the mixed formulation and recall its well-posedness.

- Section 3 and 4 are devoted to the description and the a posteriori analysis of the finite element discretizations without and with addition of a penalty term, respectively.

- The adaptivity strategy and numerical experiments are presented in Section 5. 


\section{Presentation of the model.}

As standard in the present context, Greek indices and exponents take their values in the set $\{1,2\}$ and Latin indices and exponents take their values in the set $\{1,2,3\}$. Unless otherwise specified, the summation convention for repeated indices and exponents according to this set of values is assumed (but does not apply to $h$ and $p$ ).

Let $\omega$ be a bounded connected domain of $\mathbb{R}^{2}$ with a Lipschitz-continuous boundary $\partial \omega$. We consider a shell whose midsurface is given by $S=\varphi(\bar{\omega})$ where $\varphi$ is a one-to-one mapping in $W^{2, \infty}(\omega)^{3}$ such that the two vectors

$$
a_{\alpha}(x)=\left(\partial_{\alpha} \varphi\right)(x)
$$

are linearly independent at each point $\boldsymbol{x}$ of $\bar{\omega}$. Thus,

$$
a_{3}(x)=\frac{a_{1}(x) \wedge a_{2}(x)}{\left|a_{1}(x) \wedge a_{2}(x)\right|}
$$

is the unit normal vector on the midsurface at point $\varphi(x)$. The vectors $\boldsymbol{a}_{i}(\boldsymbol{x})$ define the local covariant basis at point $\varphi(x)$. The contravariant basis $\boldsymbol{a}^{i}(\boldsymbol{x})$ is defined by the relations $\boldsymbol{a}_{i} \cdot \boldsymbol{a}^{j}=\delta_{i}^{j}$ where $\delta_{i}^{j}$ is the Kronecker symbol. In particular $\boldsymbol{a}_{3}(\boldsymbol{x})$ coincides with $\boldsymbol{a}^{3}(\boldsymbol{x})$. Note that all these vectors belong to $W^{1, \infty}(\omega)^{3}$. The first and second fundamental forms of the surface are given in covariant components by

$$
a_{\alpha \beta}=a_{\alpha} \cdot a_{\beta} \quad \text { and } \quad b_{\alpha \beta}=a_{3} \cdot \partial_{\beta} a_{\alpha} .
$$

We set $a(\boldsymbol{x})=\left|\boldsymbol{a}_{1}(\boldsymbol{x}) \wedge \boldsymbol{a}_{2}(\boldsymbol{x})\right|^{2}$ so that $\sqrt{a(\boldsymbol{x})}$ is the area element of the midsurface in the chart $\varphi$. Similarly, the length element $\ell$ on the boundary $\partial \omega$ is given by $\sqrt{a^{\alpha \beta} \tau_{\alpha} \tau_{\beta}}$, the $a^{\alpha \beta}=a^{\alpha} \cdot a^{\beta}$ being the contravariant components of the first fundamental form and $\left(\tau_{1}, \tau_{2}\right)$ being the covariant coordinates of a unit vector tangent to $\partial \omega$. We also denote by $e$ the thickness of the shell.

Let $a^{\alpha \beta \rho \sigma}$ denote the contravariant components of the elasticity tensor. We consider here the case of a homogeneous, isotropic material with Young modulus $E>0$ and Poisson ratio $\nu, 0 \leq \nu<\frac{1}{2}$, where these components are given by

$$
a^{\alpha \beta \rho \sigma}=\frac{E}{2(1+\nu)}\left(a^{\alpha \rho} a^{\beta \sigma}+a^{\alpha \sigma} a^{\beta \rho}\right)+\frac{E \nu}{1-\nu^{2}} a^{\alpha \beta} a^{\rho \sigma} .
$$

We note that each component of the elasticity tensor belongs to $L^{\infty}(\omega)$. Moreover, this tensor satisfies the usual symmetry properties and is uniformly strictly positive: There exists a positive constant $c_{0}$ such that, for all symmetric tensors $\tau=\left(\tau_{\alpha \beta}\right)$ in $\mathbb{R}^{2 \times 2}$,

$$
a^{\alpha \beta \rho \sigma}(x) \tau_{\alpha \beta} \tau_{\rho \sigma} \geq c_{0}|\tau|^{2} \quad \text { for a.e. } \boldsymbol{x} \in \omega .
$$

In this context, the covariant components of the change of metric tensor read

$$
\gamma_{\alpha \beta}(u)=\frac{1}{2}\left(\partial_{\alpha} u \cdot a_{\beta}+\partial_{\beta} u \cdot a_{\alpha}\right)
$$


the covariant components of the change of transverse shear tensor read

$$
\delta_{\alpha 3}(u, r)=\frac{1}{2}\left(\partial_{\alpha} u \cdot a_{3}+r \cdot a_{\alpha}\right),
$$

and the covariant components of the change of curvature tensor read

$$
\chi_{\alpha \beta}(\boldsymbol{u}, \boldsymbol{r})=\frac{1}{2}\left(\partial_{\alpha} \boldsymbol{u} \cdot \partial_{\beta} \boldsymbol{a}_{3}+\partial_{\beta} \boldsymbol{u} \cdot \partial_{\alpha} \boldsymbol{a}_{3}+\partial_{\alpha} \boldsymbol{r} \cdot \boldsymbol{a}_{\beta}+\partial_{\beta} \boldsymbol{r} \cdot \boldsymbol{a}_{\alpha}\right),
$$

see [7] and [10]. Note that all these quantities make sense for shells with little regularity, and are easily expressed with the Cartesian coordinates of the unknowns and geometrical data.

We assume that the boundary $\partial \omega$ of the chart domain is divided into two parts: $\gamma_{0}$ on which the shell is clamped and the complementary part $\gamma_{1}=\partial \omega \backslash \gamma_{0}$ on which the shell is subjected to applied tractions and moments. From now on, we suppose that $\gamma_{0}$ has a finite number of connected components and a strictly positive 1-dimensional measure. To take into account the boundary conditions, we define the space

$$
H_{\gamma_{0}}^{1}(\omega)=\left\{\mu \in H^{1}(\omega) ; \mu=0 \text { on } \gamma_{0}\right\} .
$$

Let us now consider the function space, introduced in [7] and [10], which is appropriate in the context of shells with little regularity

$$
\mathbb{V}(\omega)=\left\{V=(\boldsymbol{v}, s) \in H_{\gamma_{0}}^{1}(\omega)^{3} \times H_{\gamma_{0}}^{1}(\omega)^{3} ; s \cdot \boldsymbol{a}_{3}=0 \text { in } \omega\right\} .
$$

This space is endowed with the natural Hilbert norm

$$
\|V\|_{\mathbb{V}(\omega)}=\left(\|v\|_{H^{1}(\omega)^{3}}^{2}+\|s\|_{H^{1}(\omega)^{3}}^{2}\right)^{1 / 2} .
$$

We now recall the variational formulation of the problem corresponding to the linear Naghdi model for shells with little regularity. For data $(\boldsymbol{f}, \boldsymbol{N}, \boldsymbol{M})$ in $L^{2}(\omega)^{3} \times L^{2}\left(\gamma_{1}\right)^{3} \times$ $L^{2}\left(\gamma_{1}\right)^{3}$, it reads

Find $U=(\boldsymbol{u}, \boldsymbol{r})$ in $\mathbb{V}(\omega)$ such that

$$
\forall V \in \mathbb{V}(\omega), \quad a(U, V)=\mathcal{L}(V),
$$

where the bilinear form $a(\cdot, \cdot)$ is defined by

$$
\begin{aligned}
a(U, V)=\int_{\omega}\left\{e a ^ { \alpha \beta \rho \sigma } \left[\gamma_{\alpha \beta}(u) \gamma_{\rho \sigma}(\boldsymbol{v})+\right.\right. & \left.\frac{e^{2}}{12} \chi_{\alpha \beta}(U) \chi_{\rho \sigma}(V)\right] \\
& \left.+2 e \frac{E}{1+\nu} a^{\alpha \beta} \delta_{\alpha 3}(U) \delta_{\beta 3}(V)\right\} \sqrt{a} d \boldsymbol{x},
\end{aligned}
$$

and the linear form $\mathcal{L}(\cdot)$ is given by

$$
\mathcal{L}(V)=\int_{\omega} \boldsymbol{f} \cdot \boldsymbol{v} \sqrt{a} d \boldsymbol{x}+\int_{\gamma_{1}}(\boldsymbol{N} \cdot \boldsymbol{v}+\boldsymbol{M} \cdot \boldsymbol{s}) \ell d \tau .
$$


The data $f, N$ and $M$ represent a given resultant force density, an applied traction density and an applied moment density, respectively. In the above formulas, the thickness $e$ of the shell is assumed to be constant and positive.

The form $\mathcal{L}$ is clearly continuous on $\mathbb{V}(\omega)$ and its norm satisfies, with obvious notation,

$$
\|\mathcal{L}\| \leq c\left(\|f\|_{L^{2}(\omega)^{3}}+\|N\|_{L^{2}\left(\gamma_{1}\right)^{3}}+\|M\|_{L^{2}\left(\gamma_{1}\right)^{3}}\right) .
$$

We refer to [7] and [10] for the proof of the following ellipticity property: There exists a constant $c_{*}>0$ such that

$$
\forall V \in \mathbb{V}(\omega), \quad a(V, V) \geq c_{*}\|V\|_{\mathbb{V}(\omega)}^{2} .
$$

All this leads to the next statement (which is still valid for slightly less regular data, see $[3$, Thm 2.2], however we have no application for that).

Theorem 2.1. For any data $(\boldsymbol{f}, \boldsymbol{N}, \boldsymbol{M})$ in $L^{2}(\omega)^{3} \times L^{2}\left(\gamma_{1}\right)^{3} \times L^{2}\left(\gamma_{1}\right)^{3}$, problem (2.9) admits a unique solution $U$ in $\mathbb{V}(\omega)$. Moreover this solution satisfies

$$
\|U\|_{\mathbb{V}(\omega)} \leq c\|\mathcal{L}\| .
$$

In order to describe the discrete problem, we observe that the tangency constraint $\boldsymbol{s} \cdot \boldsymbol{a}_{3}=0$ which appears in the definition of $\mathbb{V}(\omega)$ clearly cannot be implemented in a standard way for a general shell. So the approach used in [8] consists in handling this constraint via the introduction of a Lagrange multiplier. Let us consider the relaxed function space

$$
\mathbb{X}(\omega)=H_{\gamma_{0}}^{1}(\omega)^{3} \times H_{\gamma_{0}}^{1}(\omega)^{3},
$$

still equipped with the norm defined in (2.8) which is now denoted by $\|\cdot\|_{\mathbb{X}(\omega)}$. We also set $\mathbb{M}(\omega)=H_{\gamma_{0}}^{1}(\omega)$. Obviously, the forms $a(\cdot, \cdot)$ and $\mathcal{L}(\cdot)$ defined in (2.10) and (2.11), respectively, are also defined (and continuous) on $\mathbb{X}(\omega) \times \mathbb{X}(\omega)$ and $\mathbb{X}(\omega)$. We consider the variational problem

Find $(U, \psi)$ in $\mathbb{X}(\omega) \times \mathbb{M}(\omega)$ such that

$$
\begin{array}{ll}
\forall V \in \mathbb{X}(\omega), & a(U, V)+b(V, \psi)=\mathcal{L}(V), \\
\forall \chi \in \mathbb{M}(\omega), & b(U, \chi)=0,
\end{array}
$$

where the bilinear form $b(\cdot, \cdot)$ is now defined by

$$
b(V, \chi)=\int_{\omega} \partial_{\alpha}\left(s \cdot a_{3}\right) \partial_{\alpha} \chi d x
$$

Since $\boldsymbol{a}_{3}$ belongs to $W^{1, \infty}(\omega)^{3}$, the form $b(\cdot, \cdot)$ is continuous on $\mathbb{X}(\omega) \times \mathbb{M}(\omega)$. Moreover, the following characterization holds

$$
\mathbb{V}(\omega)=\{V=(\boldsymbol{v}, s) \in \mathbb{X}(\omega) ; \forall \chi \in \mathbb{M}(\omega), b(V, \chi)=0\} .
$$


The following inf-sup condition on the form $b(\cdot, \cdot)$ is easily derived by taking $V=\left(\mathbf{0}, \chi \boldsymbol{a}_{3}\right)$ : There exists a positive constant $c_{\sharp}$ such that the following inf-sup condition holds

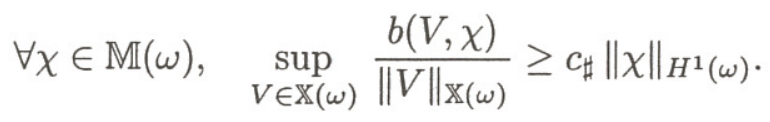

Combining all this with the ellipticity property (2.13) leads to the next result.

Theorem 2.2. For any data $(f, N, M)$ in $L^{2}(\omega)^{3} \times L^{2}\left(\gamma_{1}\right)^{3} \times L^{2}\left(\gamma_{1}\right)^{3}$, problem (2.16) admits a unique solution $(U, \psi)$ in $\mathbb{X}(\omega) \times \mathbb{M}(\omega)$. Moreover this solution satisfies

$$
\|U\|_{\mathbb{X}(\omega)}+\|\psi\|_{H^{1}(\omega)} \leq c\|\mathcal{L}\|,
$$

and its part $U$ is the solution of problem (2.9).

Remark 2.3. Since we are aiming for simplicity of implementation, we have made no attempt to make the duality term intrinsic. In fact, it does depend on the chart, whereas the other terms do not. This could arguably be considered to be a poor choice, especially if a chart is used that gives much more weight to one part of the shell compared to the rest. An intrinsic choice that obviously works is

$$
\tilde{b}(V, \chi)=\int_{\omega} a^{\alpha \beta} \partial_{\alpha}\left(s \cdot a_{3}\right) \partial_{\beta} \chi \sqrt{a} d x
$$

Remark 2.4. It can also be noted that the quantity $a(U, V)$ can be written in another form which seems more appropriate for the implementation, since it uncouples the two components $\boldsymbol{v}$ and $s$ of the test function $V$. Indeed, we introduce the contravariant components of the stress resultant

$$
n^{\rho \sigma}(u)=e a^{\alpha \beta \rho \sigma} \gamma_{\alpha \beta}(u)
$$

of the stress couple

$$
m^{\rho \sigma}(U)=\frac{e^{3}}{12} \alpha^{\alpha \beta \rho \sigma} \chi_{\alpha \beta}(U)
$$

and of the transverse shear force

$$
t^{\beta}(U)=e \frac{E}{1+\nu} a^{\alpha \beta} \delta_{\alpha 3}(U) .
$$

We also observe that

$$
\chi_{\rho \sigma}(V)=\theta_{\rho \sigma}(v)+\gamma_{\rho \sigma}(s), \quad \text { with } \quad \theta_{\rho \sigma}(v)=\frac{1}{2}\left(\partial_{\rho} v \cdot \partial_{\sigma} a_{3}+\partial_{\sigma} v \cdot \partial_{\rho} a_{3}\right)
$$

Thus, $a(U, V)$ is equal to

$$
\begin{array}{r}
a(U, V)=\int_{\omega}\left(n^{\rho \sigma}(\boldsymbol{u}) \gamma_{\rho \sigma}(\boldsymbol{v})+m^{\rho \sigma}(U) \theta_{\rho \sigma}(\boldsymbol{v})+t^{\beta}(U) \partial_{\beta} \boldsymbol{v} \cdot \boldsymbol{a}_{3}\right) \sqrt{a} d \boldsymbol{x} \\
+\int_{\omega}\left(m^{\rho \sigma}(U) \gamma_{\rho \sigma}(\boldsymbol{s})+t^{\beta}(U) s \cdot \boldsymbol{a}_{\beta}\right) \sqrt{a} d \boldsymbol{x}
\end{array}
$$


where the first integral only involves $v$ and the second one only involves $s$. By using this new form, together with the symmetry properties $n^{\rho \sigma}(\boldsymbol{u})=n^{\sigma \rho}(\boldsymbol{u})$ and $m^{\rho \sigma}(U)=m^{\sigma \rho}(U)$, it is readily checked that problem (2.16) is equivalent to the following system of partial differential equations (here, $\boldsymbol{\nu}=\left(\nu_{1}, \nu_{2}\right)$ denotes the unit outward normal vector to $\omega$ )

$$
\begin{cases}-\partial_{\rho}\left(\left(n^{\rho \sigma}(\boldsymbol{u}) \boldsymbol{a}_{\sigma}+m^{\rho \sigma}(U) \partial_{\sigma} \boldsymbol{a}_{3}+t^{\rho}(U) \boldsymbol{a}_{3}\right) \sqrt{a}\right)=\boldsymbol{f} \sqrt{a} & \text { in } \omega, \\ -\partial_{\rho}\left(m^{\rho \sigma}(U) \boldsymbol{a}_{\sigma} \sqrt{a}\right)+t^{\beta}(U) \boldsymbol{a}_{\beta} \sqrt{a}-\partial_{\rho \rho} \psi \boldsymbol{a}_{3}=0 & \text { in } \omega, \\ \boldsymbol{r} \cdot \boldsymbol{a}_{3}=0 & \text { in } \omega, \\ \boldsymbol{u}=\boldsymbol{r}=\mathbf{0} & \text { on } \gamma_{0}, \\ \psi=0 & \text { on } \gamma_{0}, \\ \nu_{\rho}\left(n^{\rho \sigma}(\boldsymbol{u}) \boldsymbol{a}_{\sigma}+m^{\rho \sigma}(U) \partial_{\sigma} \boldsymbol{a}_{3}+t^{\rho}(U) \boldsymbol{a}_{3}\right) \sqrt{a}=\boldsymbol{N} \ell & \text { on } \gamma_{1}, \\ \nu_{\rho}\left(m^{\rho \sigma}(U) \boldsymbol{a}_{\sigma} \sqrt{a}+\partial_{\rho} \psi \boldsymbol{a}_{3}\right)=M \ell & \text { on } \gamma_{1} .\end{cases}
$$

When the form $b(\cdot, \cdot)$ is replaced by the intrinsic form $\tilde{b}(\cdot, \cdot)$ defined in $(2.21)$, the term in $\psi$ in the second line of this system is replaced by $-a_{3} \partial_{\rho}\left(a^{\rho \sigma} \partial_{\sigma} \psi \sqrt{a}\right)$, hence involves the Laplace-Beltrami operator on the shell midsurface. The Lagrange multiplier acts as a normal force in the flexural equation. This force prevents pinching, which in this case would correspond to $\boldsymbol{r} \cdot \boldsymbol{a}_{3} \neq 0$. A similar classical formulation of the boundary value problem for the Koiter model in covariant components can be found in [14, Chap. VII, $\S 7.1]$ for instance.

The two discretizations that we intend to study are constructed by the Galerkin method from problem (2.16), with addition of a penalty term for the second one. Note that a stabilization term can also be added in the first line of problem (2.16); we refer to $[8, \S 5.1]$ for the analysis of the corresponding discretization that we do not consider in this work. 


\section{A posteriori analysis of the first discrete problem.}

From now on, we assume that $\omega$ is a polygon. We introduce a regular family $\left(\mathcal{T}_{h}\right)_{h}$ of triangulations of $\omega$ (by triangles), in the usual sense that:

- For each $h, \bar{\omega}$ is the union of all elements of $\mathcal{T}_{h}$;

- The intersection of two different elements of $\mathcal{T}_{h}$, if not empty, is a vertex or a whole edge of both of them;

- The ratio of the diameter $h_{K}$ of any element $K$ of $\mathcal{T}_{h}$ to the diameter of its inscribed circle is smaller than a constant $\sigma$ independent of $h$.

We make the further non restrictive assumption that $\bar{\gamma}_{0}$ is the union of whole edges of elements of $\mathcal{T}_{h}$. As is standard, $h$ denotes the maximum of the diameters $h_{K}, K \in \mathcal{T}_{h}$. In what follows, $c, c^{\prime}, \ldots$ stand for generic constants which may vary from line to line but are always independent of $h$.

For each nonnegative integer $k$ and any element $K$ of $\mathcal{T}_{h}$, let $\mathcal{P}_{k}(K)$ denote the space of restrictions to $K$ of polynomials with total degree $\leq k$. We thus define the basic discrete space

$$
\mathbb{M}_{h}=\left\{\chi_{h} \in H^{1}(\omega) ; \forall K \in \mathcal{T}_{h}, \chi_{h \mid K} \in \mathcal{P}_{1}(K)\right\},
$$

next the spaces that are involved in the discrete problem

$$
\mathbb{M}_{h}^{\gamma_{0}}=\mathbb{M}_{h} \cap H_{\gamma_{0}}^{1}(\omega), \quad \mathbb{X}_{h}=\left(\mathbb{M}_{h}^{\gamma_{0}}\right)^{3} \times\left(\mathbb{M}_{h}^{\gamma_{0}}\right)^{3}
$$

Indeed, applying the Galerkin method to (2.16) leads to the problem

Find $\left(U_{h}, \psi_{h}\right)$ in $\mathbb{X}_{h} \times \mathbb{M}_{h}^{\gamma_{0}}$ such that

$$
\begin{aligned}
& \forall V_{h} \in \mathbb{X}_{h}, \quad a\left(U_{h}, V_{h}\right)+b\left(V_{h}, \psi_{h}\right)=\mathcal{L}\left(V_{h}\right), \\
& \forall \chi_{h} \in \mathbb{M}_{h}^{\gamma_{0}}, \quad b\left(U_{h}, \chi_{h}\right)=0 .
\end{aligned}
$$

We refer to [8, Thm 5.3 \& Prop. 5.8] for the following results of a priori analysis.

Proposition 3.1. There exists $h_{0}>0$ such that, for all $h \leq h_{0}$ and for any data $(f, N, M)$ in $L^{2}(\omega)^{3} \times L^{2}\left(\gamma_{1}\right)^{3} \times L^{2}\left(\gamma_{1}\right)^{3}$, problem (3.3) admits a unique solution $\left(U_{h}, \psi_{h}\right)$ in $\mathbb{X}_{h} \times \mathbb{M}_{h}^{\gamma_{0}}$. Moreover, this solution satisfies

$$
\left\|U_{h}\right\|_{\mathbb{X}(\omega)}+\left\|\psi_{h}\right\|_{H^{1}(\omega)} \leq c\|\mathcal{L}\|
$$

and, if the solution $(U, \psi)$ of problem (2.16) belongs to $\left(H^{2}(\omega)^{3} \times H^{2}(\omega)^{3}\right) \times H^{2}(\omega)$, the following a priori error estimate holds

$$
\left\|U-U_{h}\right\|_{\mathbb{X}(\omega)}+\left\|\psi-\psi_{h}\right\|_{H^{1}(\omega)} \leq c(U, \psi) h,
$$

for a constant $c(U, \psi)$ only depending on the solution $(U, \psi)$.

The a posteriori analysis of problem (3.3) relies on the residual equations

$$
\begin{gathered}
\forall V \in \mathbb{X}(\omega), \forall V_{h} \in \mathbb{X}_{h}, \quad a\left(U-U_{h}, V\right)+b\left(V, \psi-\psi_{h}\right) \\
=\mathcal{L}\left(V-V_{h}\right)-a\left(U_{h}, V-V_{h}\right)-b\left(V-V_{h}, \psi_{h}\right), \\
\forall \chi \in \mathbb{M}(\omega), \forall \chi_{h} \in \mathbb{M}_{h}^{\gamma_{0}}, \quad b\left(U-U_{h}, \chi\right)=-b\left(U_{h}, \chi-\chi_{h}\right) .
\end{gathered}
$$


As usual for this type of problem, see [22, Chap. 3], the construction of error indicators from these equations requires approximations of the data and of the coefficients.

Approximation of THE DATA. Let $\mathcal{E}_{h}^{1}$ denote the set of edges of elements of $\mathcal{T}_{h}$ which are contained in $\bar{\gamma}_{1}$. From now on, we consider an approximation $f_{h}$ of $f$ in $\mathbb{Z}_{h}$ and approximations $N_{h}$ and $M_{h}$ of $N$ and $M$ in $\mathbb{Z}_{h}^{1}$, where the spaces $\mathbb{Z}_{h}$ and $\mathbb{Z}_{h}^{1}$ are defined by

$$
\begin{aligned}
\mathbb{Z}_{h}=\left\{\boldsymbol{g}_{h} \in L^{2}(\omega)^{3} ; \forall K\right. & \left.\in \mathcal{T}_{h}, \boldsymbol{g}_{h \mid K} \in \mathcal{P}_{0}(K)^{3}\right\}, \\
\mathbb{Z}_{h}^{1} & =\left\{\boldsymbol{P}_{h} \in L^{2}\left(\gamma_{1}\right)^{3} ; \forall e \in \mathcal{E}_{h}^{1}, \boldsymbol{P}_{h \mid e} \in \mathcal{P}_{0}(e)^{3}\right\} .
\end{aligned}
$$

Approximation of THE COEFFicients. For reasons that will appear later on, we introduce approximations of the scalar coefficients $a^{\alpha \beta}, a^{\alpha \beta \rho \sigma}, \sqrt{a}$ and $\ell$ in the space $\mathbb{M}_{h}$, that we denote by $a_{h}^{\alpha \beta}, a_{h}^{\alpha \beta \rho \sigma},(\sqrt{a})_{h}$ and $\ell_{h}$, respectively. We make the further assumption (which is usually satisfied for Lagrange finite elements, see [6, Th. IX.1.5] for instance) that, if $g$ denotes any of these quantities and $g_{h}$ its approximation,

$$
\left\|g_{h}\right\|_{L^{\infty}(\Omega)} \leq c\|g\|_{W^{1, \infty}(\Omega)} .
$$

Similarly, we consider approximations $\boldsymbol{a}_{k}^{h}$ of the vectors $\boldsymbol{a}_{k}$ and $\boldsymbol{d}_{\alpha}^{h}$ of the $\partial_{\alpha} \boldsymbol{a}_{3}$ in the space $\left(\mathbb{M}_{h}\right)^{3}$. We also agree to denote by $\gamma_{\alpha \beta}^{h}(\cdot), \delta_{\alpha 3}^{h}(\cdot)$ and $\chi_{\alpha \beta}^{h}(\cdot)$ the components of the tensors introduced in (2.3) to (2.5) where all coefficients are replaced by their approximations. For instance, $\gamma_{\alpha \beta}^{h}(\cdot)$ is given by

$$
\gamma_{\alpha \beta}^{h}(\boldsymbol{u})=\frac{1}{2}\left(\partial_{\alpha} \boldsymbol{u} \cdot \boldsymbol{a}_{\beta}^{h}+\partial_{\beta} \boldsymbol{u} \cdot \boldsymbol{a}_{\alpha}^{h}\right)
$$

This leads to the definition of an approximate linear form

$$
\mathcal{L}_{h}(V)=\int_{\omega} f_{h} \cdot \boldsymbol{v}(\sqrt{a})_{h} d \boldsymbol{x}+\int_{\gamma_{1}}\left(N_{h} \cdot \boldsymbol{v}+M_{h} \cdot s\right) \ell_{h} d \tau .
$$

and also of approximate bilinear forms

$$
\begin{aligned}
a_{h}(U, V)= & \int_{\omega}\left\{e a _ { h } ^ { \alpha \beta \rho \sigma } \left[\gamma_{\alpha \beta}^{h}(\boldsymbol{u}) \gamma_{\rho \sigma}^{h}(\boldsymbol{v})\right.\right. \\
+ & \left.+\frac{e^{2}}{12} \chi_{\alpha \beta}^{h}(U) \chi_{\rho \sigma}^{h}(V)\right] \\
& \left.+2 e \frac{E}{1+\nu} a_{h}^{\alpha \beta} \delta_{\alpha 3}^{h}(U) \delta_{\beta 3}^{h}(V)\right\}(\sqrt{a})_{h} d \boldsymbol{x} \\
b_{h}(V, \chi)= & \int_{\omega}\left(\partial_{\alpha} s \cdot \boldsymbol{a}_{3}^{h}+s \cdot \boldsymbol{d}_{\alpha}^{h}\right) \partial_{\alpha} \chi d x
\end{aligned}
$$

To go further, we recall some standard notation.

Notation 3.2. For each element $K$ of $\mathcal{T}_{h}$,

(i) $\mathcal{E}_{K}$ denotes the set of edges of $K$ which are not contained in $\bar{\gamma}_{0}$ and $\mathcal{E}_{K}^{1}$ the set of elements of $\mathcal{E}_{K}$ which are contained in $\bar{\gamma}_{1}$;

(ii) for each $e$ in $\mathcal{E}_{K}, \nu=\left(\nu_{1}, \nu_{2}\right)$ is a unit vector normal to $e$, with the further assumption 
that, when $e$ belongs to $\mathcal{E}_{K}^{1}, \nu$ is outward to $\omega$;

(iii) for each $e$ in $\mathcal{E}_{K}, h_{e}$ stands for the length of $e$;

(iv) for each $e$ in $\mathcal{E}_{K} \backslash \mathcal{E}_{K}^{1},[\cdot]_{e}$ denotes the jump through $e$ (making its sign precise is needless);

(v) $\omega_{K}$ is the union of triangles of $\mathcal{T}_{h}$ that share an edge with $K$;

(vi) $\Delta_{K}$ is the union of triangles of $\mathcal{T}_{h}$ that intersect $K$.

We also recall from [6, Th. IX.3.11 \& Cor. IX.3.12] the existence of a Clément type operator $\mathcal{R}_{h}$ which maps $H_{\gamma_{0}}^{1}(\omega)$ into $\mathbb{M}_{h}^{\gamma_{0}}$ and satisfies, for all functions $\chi$ in $H_{\gamma_{0}}^{1}(\omega)$, each $K$ in $\mathcal{T}_{h}$ and each edge $e$ of $K$ which is not contained in $\gamma_{0}$,

$$
\begin{gathered}
\left\|\chi-\mathcal{R}_{h} \chi\right\|_{L^{2}(K)}+h_{K}\left|\chi-\mathcal{R}_{h} \chi\right|_{H^{1}(K)} \leq c h_{K}\|\chi\|_{H^{1}\left(\Delta_{K}\right)}, \\
\left\|\chi-\mathcal{R}_{h} \chi\right\|_{L^{2}(e)} \leq c h_{e}^{\frac{1}{2}}\|\chi\|_{H^{1}\left(\Delta_{K}\right)} .
\end{gathered}
$$

Indeed, the idea is now to take $V_{h}$ equal to $\left(\mathcal{R}_{h} v, \mathcal{R}_{h} s\right)$ and $\chi_{h}$ equal to $\mathcal{R}_{h} \chi$ in (3.6).

The next three lemmas are devoted to the evaluation of the error stemming from the approximation of the data and the coefficients.

Lemma 3.3. The following estimate holds for any $V$ in $\mathbb{X}(\omega)$ and with $V_{h}=\left(\mathcal{R}_{h} v, \mathcal{R}_{h} s\right)$,

$$
\begin{aligned}
& \left|\left(\mathcal{L}-\mathcal{L}_{h}\right)\left(V-V_{h}\right)\right| \\
& \leq c\left(\left(\sum_{K \in \mathcal{T}_{h}}\left(h_{K}^{2}\left\|f-f_{h}\right\|_{L^{2}(K)^{3}}^{2}+\sum_{e \in \mathcal{E}_{K}^{1}} h_{e}\left(\left\|N-N_{h}\right\|_{L^{2}(e)^{3}}^{2}+\left\|M-M_{h}\right\|_{L^{2}(e)^{3}}^{2}\right)\right)^{\frac{1}{2}}\right.\right. \\
& \left.\quad+\left(h\left\|\sqrt{a}-(\sqrt{a})_{h}\right\|_{L^{\infty}(\omega)}+h^{\frac{1}{2}}\left\|\ell-\ell_{h}\right\|_{L^{\infty}\left(\gamma_{1}\right)}\right)\|\mathcal{L}\|\right)\|V\|_{\mathbb{X}(\omega)} .
\end{aligned}
$$

Proof: We have

$$
\begin{aligned}
& \left(\mathcal{L}-\mathcal{L}_{h}\right)\left(V-V_{h}\right) \\
& =\int_{\omega} \boldsymbol{f} \cdot\left(\boldsymbol{v}-\mathcal{R}_{h} \boldsymbol{v}\right)\left(\sqrt{a}-(\sqrt{a})_{h}\right) d \boldsymbol{x}+\int_{\omega}\left(\boldsymbol{f}-\boldsymbol{f}_{h}\right) \cdot\left(\boldsymbol{v}-\mathcal{R}_{h} \boldsymbol{v}\right)(\sqrt{a})_{h} d \boldsymbol{x} \\
& \quad+\int_{\gamma_{1}}\left(N \cdot\left(\boldsymbol{v}-\mathcal{R}_{h} \boldsymbol{v}\right)+M \cdot\left(s-\mathcal{R}_{h} s\right)\right)\left(\ell-\ell_{h}\right) d \tau \\
& \quad+\int_{\gamma_{1}}\left(N-N_{h}\right) \cdot\left(\boldsymbol{v}-\mathcal{R}_{h} \boldsymbol{v}\right) \ell_{h} d \tau+\int_{\gamma_{1}}\left(M-M_{h}\right) \cdot\left(s-\mathcal{R}_{h} s\right) \ell_{h} d \tau
\end{aligned}
$$

So the desired estimate follows from Cauchy-Schwarz inequalities combined with (3.8) and (3.12).

Lemma 3.4. The following estimate holds for any $V$ in $\mathbb{X}(\omega)$ and with $V_{h}=\left(\mathcal{R}_{h} v, \mathcal{R}_{h} s\right)$,

$$
\begin{aligned}
& \left|\left(a-a_{h}\right)\left(U_{h}, V-V_{h}\right)\right| \leq c\left(\left\|\sqrt{a}-(\sqrt{a})_{h}\right\|_{L^{\infty}(\omega)}\right. \\
& \quad+\sup _{1 \leq \alpha, \beta, \rho, \sigma \leq 2}\left\|a^{\alpha \beta \rho \sigma}-a_{h}^{\alpha \beta \rho \sigma}\right\|_{L^{\infty}(\omega)}+\sup _{1 \leq \alpha, \beta \leq 2}\left\|a^{\alpha \beta}-a_{h}^{\alpha \beta}\right\|_{L^{\infty}(\omega)} \\
& \left.\quad+\sup _{1 \leq k \leq 3}\left\|a_{k}-\boldsymbol{a}_{k}^{h}\right\|_{L^{\infty}(\omega)^{3}}+\sup _{1 \leq \alpha \leq 2}\left\|\partial_{\alpha} a_{3}-\boldsymbol{d}_{\alpha}^{h}\right\|_{L^{\infty}(\omega)^{3}}\right)\|\mathcal{L}\|\|V\|_{\mathbb{X}(\omega)} .
\end{aligned}
$$


Proof: We only give an abridged proof of this technical result. We use estimate (3.4) and observe from (3.12) that $\left\|V-V_{h}\right\|_{\mathbb{X}(\omega)}$ is bounded by a constant times $\|V\|_{\mathbb{X}(\omega)}$. Next:

1) By the same arguments as in the previous proof and (3.8), the difference between $a\left(U_{h}, V-V_{h}\right)$ and this same form with the coefficients $\sqrt{a}, a^{\alpha \beta \rho \sigma}$ and $a^{\alpha \beta}$ replaced by their approximations is bounded by

$$
\begin{aligned}
c\left(\left\|\sqrt{a}-(\sqrt{a})_{h}\right\|_{L^{\infty}(\omega)}+\sup _{1 \leq \alpha, \beta, \rho, \sigma \leq 2} \|\right. & a^{\alpha \beta \rho \sigma}-a_{h}^{\alpha \beta \rho \sigma} \|_{L^{\infty}(\omega)} \\
& \left.+\sup _{1 \leq \alpha, \beta \leq 2}\left\|a^{\alpha \beta}-a_{h}^{\alpha \beta}\right\|_{L^{\infty}(\omega)}\right)\|\mathcal{L}\|\|V\|_{\mathbb{X}(\omega) .}
\end{aligned}
$$

2) We use the triangle inequality

$\left\|\gamma_{\alpha \beta}\left(\boldsymbol{u}_{h}\right)-\gamma_{\alpha \beta}^{h}\left(\boldsymbol{u}_{h}\right)\right\|_{L^{2}(\omega)} \leq\left\|\partial_{\alpha} \boldsymbol{u}_{h}\right\|_{L^{2}(\omega)^{3}}\left\|\boldsymbol{a}_{\beta}-\boldsymbol{a}_{\beta}^{h}\right\|_{L^{\infty}(\omega)^{3}}+\left\|\partial_{\beta} \boldsymbol{u}_{h}\right\|_{L^{2}(\Omega)^{3}}\left\|\boldsymbol{a}_{\alpha}-\boldsymbol{a}_{\alpha}^{h}\right\|_{L^{\infty}(\omega)^{3}}$, and similar ones with $\boldsymbol{u}_{h}$ replaced by $\boldsymbol{v}-\boldsymbol{v}_{h}, \gamma_{\alpha \beta}\left(\boldsymbol{u}_{h}\right)$ replaced by $\chi_{\alpha \beta}\left(U_{h}\right)$ and so on. Combining all this and using (3.4) lead to the desired estimate.

Lemma 3.5. The following estimates hold

(i) for any $V$ in $\mathbb{X}(\omega)$ and with $V_{h}=\left(\mathcal{R}_{h} v, \mathcal{R}_{h} s\right)$,

$$
\left|\left(b-b_{h}\right)\left(V-V_{h}, \psi_{h}\right)\right| \leq c\left(\left\|a_{3}-a_{3}^{h}\right\|_{L^{\infty}(\omega)^{3}}+\left\|\partial_{\alpha} a_{3}-d_{\alpha}^{h}\right\|_{L^{\infty}(\omega)^{3}}\right)\|\mathcal{L}\|\|V\|_{\mathbb{X}(\omega)},
$$

(ii) for any $\chi$ in $\mathbb{M}(\omega)$ and with $\chi_{h}=\mathcal{R}_{h} \chi$,

$$
\left|\left(b-b_{h}\right)\left(U_{h}, \chi-\chi_{h}\right)\right| \leq c\left(\left\|a_{3}-a_{3}^{h}\right\|_{L^{\infty}(\omega)^{3}}+\left\|\partial_{\alpha} a_{3}-d_{\alpha}^{h}\right\|_{L^{\infty}(\omega)^{3}}\right)\|\mathcal{L}\|\|\chi\|_{H^{1}(\omega)} .
$$

Proof: Owing to the definition $(3.11)$ of $b_{h}(\cdot, \cdot)$, we have the expansion

$$
\left(b-b_{h}\right)(V, \chi)=\int_{\omega}\left(\partial_{\alpha} s \cdot\left(a_{3}-a_{3}^{h}\right)+s \cdot\left(\partial_{\alpha} a_{3}-d_{\alpha}^{h}\right)\right) \partial_{\alpha} \chi d x .
$$

Combining this with (3.4) yields (3.15) and (3.16).

From these lemmas, we now define the quantities linked to the local approximation error on the data: For each $K$ in $\mathcal{T}_{h}$,

$$
\varepsilon_{K}^{(d)}=h_{K}\left\|f-f_{h}\right\|_{L^{2}(K)^{3}}+\sum_{e \in \mathcal{E}_{K}^{1}} h_{e}^{\frac{1}{2}}\left(\left\|N-N_{h}\right\|_{L^{2}(e)^{3}}+\left\|M-M_{h}\right\|_{L^{2}(e)^{3}}\right),
$$

and also to the global approximation error on the coefficients:

$$
\begin{gathered}
\varepsilon_{h}^{(c)}=\left(\left\|\sqrt{a}-(\sqrt{a})_{h}\right\|_{L^{\infty}(\omega)}+h^{\frac{1}{2}}\left\|\ell-\ell_{h}\right\|_{L^{\infty}\left(\gamma_{1}\right)}+\sup _{1 \leq \alpha, \beta, \rho, \sigma \leq 2}\left\|a^{\alpha \beta \rho \sigma}-a_{h}^{\alpha \beta \rho \sigma}\right\|_{L^{\infty}(\omega)}\right. \\
+\sup _{1 \leq \alpha, \beta \leq 2}\left\|a^{\alpha \beta}-a_{h}^{\alpha \beta}\right\|_{L^{\infty}(\omega)}+\sup _{1 \leq k \leq 3}\left\|a_{k}-a_{k}^{h}\right\|_{L^{\infty}(\omega)^{3}} \\
\left.+\sup _{1 \leq \alpha \leq 2}\left\|\partial_{\alpha} \boldsymbol{a}_{3}-d_{\alpha}^{h}\right\|_{L^{\infty}(\omega)^{3}}\right)\|\mathcal{L}\| .
\end{gathered}
$$


We are now in a position to prove the a posteriori error estimate. In order to state it, we introduce the error indicators. For simplicity, we use Remark 2.4 to write $a(U, V)$ and observe that a similar form holds for $a_{h}(U, V)$, with obvious notation for the quantities $n_{h}^{\rho \sigma}(\cdot), m_{h}^{\rho \sigma}(\cdot), t_{h}^{\beta}(\cdot)$ and $\theta_{\rho \sigma}^{h}(\cdot)$ (in comparison with (2.22) to (2.25), all coefficients are replaced by their approximations). For each $K$ in $\mathcal{T}_{h}$, the error indicator $\eta_{K}$ is defined by

$$
\eta_{K}=\eta_{K 1}+\eta_{K 2}+\eta_{K 3}
$$

with

$$
\begin{aligned}
& \eta_{K 1}=h_{K}\left\|f_{h}(\sqrt{a})_{h}+\partial_{\rho}\left(\left(n_{h}^{\rho \sigma}\left(u_{h}\right) a_{\sigma}^{h}+m_{h}^{\rho \sigma}\left(U_{h}\right) d_{\sigma}^{h}+t_{h}^{\rho}\left(U_{h}\right) a_{3}^{h}\right)(\sqrt{a})_{h}\right)\right\|_{L^{2}(K)^{3}} \\
& +\sum_{e \in \mathcal{E}_{K} \backslash \mathcal{E}_{K}^{1}} h_{e}^{\frac{1}{2}}\left\|\left[\nu_{\rho}\left(n_{h}^{\rho \sigma}\left(\boldsymbol{u}_{h}\right) \boldsymbol{a}_{\sigma}^{h}+m_{h}^{\rho \sigma}\left(U_{h}\right) d_{\sigma}^{h}+t_{h}^{\rho}\left(U_{h}\right) \boldsymbol{a}_{3}^{h}\right)(\sqrt{a})_{h}\right]_{e}\right\|_{L^{2}(e)^{3}} \\
& +\sum_{e \in \mathcal{E}_{K}^{1}} h_{e}^{\frac{1}{2}}\left\|N_{h} \ell_{h}-\nu_{\rho}\left(n_{h}^{\rho \sigma}\left(\boldsymbol{u}_{h}\right) \boldsymbol{a}_{\sigma}^{h}+m_{h}^{\rho \sigma}\left(U_{h}\right) \boldsymbol{d}_{\sigma}^{h}+t_{h}^{\rho}\left(U_{h}\right) \boldsymbol{a}_{3}^{h}\right)(\sqrt{a})_{h}\right\|_{L^{2}(e)^{3}}, \\
& \eta_{K 2}=h_{K}\left\|\partial_{\rho}\left(m_{h}^{\rho \sigma}\left(U_{h}\right) \boldsymbol{a}_{\sigma}^{h}(\sqrt{a})_{h}\right)-t_{h}^{\beta}\left(U_{h}\right) \boldsymbol{a}_{\beta}^{h}(\sqrt{a})_{h}+\partial_{\rho}\left(a_{3}^{h} \partial_{\rho} \psi_{h}\right)-d_{\rho}^{h} \partial_{\rho} \psi_{h}\right\|_{L^{2}(K)^{3}} \\
& +\sum_{e \in \mathcal{E}_{K} \backslash \mathcal{E}_{K}^{1}} h_{e}^{\frac{1}{2}}\left\|\left[\nu_{\rho} m_{h}^{\rho \sigma}\left(U_{h}\right) a_{\sigma}^{h}(\sqrt{a})_{h}+\nu_{\rho} \partial_{\rho} \psi_{h} a_{3}^{h}\right]_{e}\right\|_{L^{2}(e)^{3}} \\
& +\sum_{e \in \mathcal{E}_{K}^{1}} h_{e}^{\frac{1}{2}}\left\|M_{h} \ell_{h}-\nu_{\rho} m_{h}^{\rho \sigma}\left(U_{h}\right) a_{\sigma}^{h}(\sqrt{a})_{h}-\nu_{\rho} \partial_{\rho} \psi_{h} a_{3}^{h}\right\|_{L^{2}(e)^{3}}, \\
& \eta_{K 3}=h_{K}\left\|\partial_{\alpha}\left(\partial_{\alpha} \boldsymbol{r}_{h} \cdot \boldsymbol{a}_{3}^{h}+\boldsymbol{r}_{h} \cdot \boldsymbol{d}_{\alpha}^{h}\right)\right\|_{L^{2}(K)} \\
& +\sum_{e \in \mathcal{E}_{K} \backslash \mathcal{E}_{K}^{1}} h_{e}^{\frac{1}{2}}\left\|\left[\partial_{\nu}\left(\boldsymbol{r}_{h} \cdot \boldsymbol{a}_{3}^{h}\right)\right]_{e}\right\|_{L^{2}(e)}+\sum_{e \in \mathcal{E}_{K}^{1}} h_{e}^{\frac{1}{2}}\left\|\partial_{\nu}\left(\boldsymbol{r}_{h} \cdot \boldsymbol{a}_{3}^{h}\right)\right\|_{L^{2}(e)} .
\end{aligned}
$$

Note that these indicators are easy to compute since they only involve polynomial functions. All of them are of residual type: Indeed, when suppressing all the indices and exponents $h$ in their definitions and looking at system (2.27), we observe that they cancel.

Theorem 3.6. For any data $(\boldsymbol{f}, \boldsymbol{N}, \boldsymbol{M})$ in $L^{2}(\omega)^{3} \times L^{2}\left(\gamma_{1}\right)^{3} \times L^{2}\left(\gamma_{1}\right)^{3}$, the following a posteriori error estimate holds between the solution $(U, \psi)$ of problem (2.16) and the solution $\left(U_{h}, \psi_{h}\right)$ of problem (3.3)

$$
\left\|U-U_{h}\right\|_{\mathbb{X}(\omega)}+\left\|\psi-\psi_{h}\right\|_{H^{1}(\omega)} \leq c\left(\left(\sum_{K \in \mathcal{T}_{h}}\left(\eta_{K}^{2}+\varepsilon_{K}^{(d) 2}\right)\right)^{\frac{1}{2}}+\varepsilon_{h}^{(c)}\right) .
$$

Proof: Standard arguments combined with the ellipticity property (2.13) and the inf-sup condition (2.19) yield that the error is bounded by a constant times the norm in $\mathbb{X}(\omega)^{\prime}$ of the right-hand side of the first line in (3.6) plus the norm in $\mathbb{M}(\omega)^{\prime}$ of the right-hand side of the second line. We then use triangle inequalities combined with Lemmas 3.3 to 3.5 to replace $\mathcal{L}\left(V-V_{h}\right)$ by $\mathcal{L}_{h}\left(V-V_{h}\right), a\left(U_{h}, V-V_{h}\right)$ by $a_{h}\left(U_{h}, V-V_{h}\right)$ and the same for the 
two terms involving the form $b(\cdot, \cdot)$. So it remains to bound the three quantities

$$
\begin{gathered}
A_{1}=\sup _{\boldsymbol{v} \in H_{\gamma_{0}}^{1}(\omega)^{3}} \frac{\mathcal{L}_{h}\left(\boldsymbol{v}-\mathcal{R}_{h} \boldsymbol{v}, \mathbf{0}\right)-a_{h}\left(U_{h},\left(\boldsymbol{v}-\mathcal{R}_{h} \boldsymbol{v}, \mathbf{0}\right)\right)}{\|\boldsymbol{v}\|_{H^{1}(\omega)^{3}}}, \\
A_{2}=\sup _{s \in H_{\gamma_{0}}^{1}(\omega)^{3}} \frac{\mathcal{L}_{h}\left(\mathbf{0}, s-\mathcal{R}_{h} s\right)-a_{h}\left(U_{h},\left(\mathbf{0}, \boldsymbol{s}-\mathcal{R}_{h} s\right)\right)-b_{h}\left(\left(\mathbf{0}, s-\mathcal{R}_{h} s\right), \psi_{h}\right)}{\|s\|_{H^{1}(\omega)^{3}},} \\
A_{3}=\sup _{\chi \in \mathbb{M}(\omega)} \frac{b_{h}\left(U_{h}, \chi-\mathcal{R}_{h} \chi\right)}{\|\chi\|_{H^{1}(\omega)}} .
\end{gathered}
$$

(note indeed that $b_{h}\left((\boldsymbol{v}, \mathbf{0}), \psi_{h}\right)$ is zero).

1) Setting $\boldsymbol{w}=\boldsymbol{v}-\mathcal{R}_{h} \boldsymbol{v}$ and using once more the symmetry properties of the $n_{h}^{\rho \sigma}(\cdot)$ and $m_{h}^{\rho \sigma}(\cdot)$, we have

$$
\begin{aligned}
\mathcal{L}_{h}(\boldsymbol{v} & \left.-\mathcal{R}_{h} \boldsymbol{v}, \mathbf{0}\right)-a_{h}\left(U_{h},\left(\boldsymbol{v}-\mathcal{R}_{h} \boldsymbol{v}, \mathbf{0}\right)\right)=\int_{\omega} \boldsymbol{f}_{h} \cdot \boldsymbol{w}(\sqrt{a})_{h} d \boldsymbol{x}+\int_{\gamma_{1}} \boldsymbol{N}_{h} \cdot \boldsymbol{w} \ell_{h} d \tau \\
& \left.-\int_{\omega}\left(n_{h}^{\rho \sigma}\left(\boldsymbol{u}_{h}\right) \partial_{\rho} \boldsymbol{w} \cdot \boldsymbol{a}_{\sigma}^{h}+m_{h}^{\rho \sigma}\left(U_{h}\right) \partial_{\rho} \boldsymbol{w} \cdot \boldsymbol{d}_{\sigma}^{h}\right)+t_{h}^{\beta}\left(U_{h}\right) \partial_{\beta} \boldsymbol{w} \cdot \boldsymbol{a}_{3}^{h}\right)(\sqrt{a})_{h} d \boldsymbol{x}
\end{aligned}
$$

By cutting the integrals on $\omega$ into the sum of integrals on the $K$ in $\mathcal{T}_{h}$ and integrating by parts on each $K$, we derive

$$
\begin{aligned}
\mathcal{L}_{h}\left(\boldsymbol{v}-\mathcal{R}_{h} \boldsymbol{v}, \mathbf{0}\right)-a_{h}\left(U_{h},\left(\boldsymbol{v}-\mathcal{R}_{h} \boldsymbol{v}, \mathbf{0}\right)\right)=\int_{\omega} \boldsymbol{f}_{h} \cdot \boldsymbol{w}(\sqrt{a})_{h} d \boldsymbol{x}+\int_{\gamma_{1}} \boldsymbol{N}_{h} \cdot \boldsymbol{w} \ell_{h} d \tau \\
+\sum_{K \in \mathcal{T}_{h}}\left(\int_{K} \partial_{\rho}\left(\left(n_{h}^{\rho \sigma}\left(\boldsymbol{u}_{h}\right) \boldsymbol{a}_{\sigma}^{h}+m_{h}^{\rho \sigma}\left(U_{h}\right) \boldsymbol{d}_{\sigma}^{h}+t_{h}^{\rho}\left(U_{h}\right) \boldsymbol{a}_{3}^{h}\right)(\sqrt{a})_{h}\right) \cdot \boldsymbol{w} d \boldsymbol{x}\right. \\
\left.-\int_{\partial K} \nu_{\rho}\left(n_{h}^{\rho \sigma}\left(\boldsymbol{u}_{h}\right) \boldsymbol{a}_{\sigma}^{h}+m_{h}^{\rho \sigma}\left(U_{h}\right) \boldsymbol{d}_{\sigma}^{h}+t_{h}^{\rho}\left(U_{h}\right) \boldsymbol{a}_{3}^{h}\right)(\sqrt{a})_{h} \cdot \boldsymbol{w} d \tau\right)
\end{aligned}
$$

Using Cauchy-Schwarz inequalities combined with (3.12) leads to

$$
A_{1} \leq c\left(\sum_{K \in \mathcal{T}_{h}} \eta_{K 1}^{2}\right)^{\frac{1}{2}}
$$

2) Setting $t=s-\mathcal{R}_{h} s$, we also have

$$
\begin{array}{r}
\mathcal{L}_{h}\left(\mathbf{0}, s-\mathcal{R}_{h} s\right)-a_{h}\left(U_{h},\left(\mathbf{0}, s-\mathcal{R}_{h} s\right)\right)-b_{h}\left(\left(\mathbf{0}, s-\mathcal{R}_{h} s\right), \psi_{h}\right) \\
=\int_{\gamma_{1}} M_{h} \cdot \boldsymbol{t} \ell_{h} d \tau-\int_{\omega}\left(m_{h}^{\rho \sigma}\left(U_{h}\right) \partial_{\rho} \boldsymbol{t} \cdot \boldsymbol{a}_{\sigma}^{h}+t_{h}^{\beta}\left(U_{h}\right) \boldsymbol{t} \cdot \boldsymbol{a}_{\beta}^{h}\right)(\sqrt{a})_{h} d \boldsymbol{x} \\
-\int_{\omega}\left(\partial_{\alpha} \boldsymbol{t} \cdot \boldsymbol{a}_{3}^{h}+\boldsymbol{t} \cdot \boldsymbol{d}_{\alpha}^{h}\right) \partial_{\alpha} \psi_{h} d \boldsymbol{x}
\end{array}
$$

The same integrations by parts as previously and (3.12) thus lead to

$$
A_{2} \leq c\left(\sum_{K \in \mathcal{T}_{h}} \eta_{K 2}^{2}\right)^{\frac{1}{2}}
$$


3) Finally, deriving that $A_{3} \leq c\left(\sum_{K \in \mathcal{T}_{h}} \eta_{K 3}^{2}\right)^{\frac{1}{2}}$ is obvious when compared with the two previous steps.

As is standard, see $[22, \S 1.2]$, the converse estimate relies on inverse inequalities and involve local norms defined in an obvious way by restriction.

Theorem 3.7. For any data $(\boldsymbol{f}, \boldsymbol{N}, \boldsymbol{M})$ in $L^{2}(\omega)^{3} \times L^{2}\left(\gamma_{1}\right)^{3} \times L^{2}\left(\gamma_{1}\right)^{3}$, the following bound holds for all indicators $\eta_{K}$ defined in (3.19) - (3.22),

$$
\eta_{K} \leq c\left(\left\|U-U_{h}\right\|_{\mathbb{X}\left(\omega_{K}\right)}+\left\|\psi-\psi_{h}\right\|_{H^{1}\left(\omega_{K}\right)}+\left(\sum_{\kappa \subset \omega_{K}} \varepsilon_{\kappa}^{(d) 2}\right)^{\frac{1}{2}}+\varepsilon_{h}^{(c)}\right) .
$$

Proof: We only give an abridged proof of the estimate for $\eta_{K 1}$. We first write it in the more compact form

$$
\eta_{K 1}=h_{K}\left\|\boldsymbol{F}_{h}\right\|_{L^{2}(K)^{3}}+\sum_{e \in \mathcal{E}_{K} \backslash \mathcal{E}_{K}^{1}} h_{e}^{\frac{1}{2}}\left\|\left[G_{h}\right]_{e}\right\|_{L^{2}(e)^{3}}+\sum_{e \in \mathcal{E}_{K}^{1}} h_{e}^{\frac{1}{2}}\left\|N_{h} \ell_{h}-G_{h}\right\|_{L^{2}(e)^{3}} .
$$

Next, we observe that equation (3.24) still holds with $\mathcal{R}_{h} v$ replaced by $\mathbf{0}$ and we first take in this equation $\boldsymbol{v}$ equal to

$$
\boldsymbol{v}= \begin{cases}\boldsymbol{F}_{h} \psi_{K} & \text { on } K \\ \mathbf{0} & \text { on } \omega \backslash K,\end{cases}
$$

where $\psi_{K}$ denotes the bubble function on $K$. Thus, all the terms in the right-hand side of (3.24) vanish but the integral on $K$. Now, since $\boldsymbol{F}_{h}$ is a polynomial of degree $\leq 3$, appropriate inverse inequalities [22, Lemma 3.3] combined with Lemmas 3.3 to 3.5 lead to

$$
h_{K}\left\|\boldsymbol{F}_{h}\right\|_{L^{2}(K)^{3}} \leq c\left(\left\|U-U_{h}\right\|_{\mathbb{X}(K)}+\varepsilon_{K}^{(d)}+\varepsilon_{h}^{(c)}\right) .
$$

Similarly, for any edge $e$ shared by two elements $K$ and $K^{\prime}$, we take $v$ in (3.24) equal to

$$
\boldsymbol{v}= \begin{cases}\mathcal{L}_{e, \kappa}\left(\left[\boldsymbol{G}_{h}\right]_{e} \psi_{e}\right) & \text { on } \kappa \in\left\{K, K^{\prime}\right\} \\ \mathbf{0} & \text { on } \omega \backslash\left(K \cup K^{\prime}\right)\end{cases}
$$

where $\psi_{e}$ is the bubble function on $e$ and $\mathcal{L}_{e, \kappa}$ is a lifting operator from polynomials on $e$ vanishing on $\partial e$ into polynomials on $K$ vanishing on $\partial K \backslash e$ constructed by affine transformation from a fixed lifting operator on the reference triangle. This leads to a bound for the second term of $\eta_{K 1}$. Finally, for each $e$ in $\mathcal{E}_{K}^{1}$, we take $\boldsymbol{v}$ in (3.24) equal to

$$
\boldsymbol{v}= \begin{cases}\mathcal{L}_{e, \kappa}\left(\left(N_{h} \ell_{h}-G_{h}\right) \psi_{e}\right) & \text { on } K, \\ 0 & \text { on } \omega \backslash K,\end{cases}
$$

and this gives the bound for $\eta_{K 1}$.

It follows from Theorems 3.6 and 3.7 that, up to the terms $\varepsilon_{K}^{(d)}$ and $\varepsilon_{h}^{(c)}$, the full error is equivalent to the $\left(\sum_{K \in \mathcal{T}_{h}} \eta_{K}^{2}\right)^{\frac{1}{2}}$. So the estimates that we have proved are fully optimal. Moreover the quantities $\varepsilon_{K}^{(d)}$ for smooth data and $\varepsilon_{h}^{(c)}$ for a regular shell are most often negligible. Finally, estimate (3.25) is local, up to the term $\varepsilon_{h}^{(c)}$ (and this is only for simplicity). So it can be thought that the $\eta_{K}$ provide a good representation of the local error and thus form an efficient tool for adapting the mesh. 


\section{A posteriori analysis of the second discrete problem.}

We keep the notation of the previous section. Following now the approach in $[8, \S 5.1]$, we introduce a penalty parameter $\varepsilon_{p}, 0<\varepsilon_{p} \leq 1$, and define the bilinear form $c(\cdot, \cdot)$ by

$$
c(\psi, \chi)=\int_{\omega} \partial_{\alpha} \psi \partial_{\alpha} \chi d x
$$

Thus we consider the following discrete problem, constructed by adding to (3.3) a penalization term

Find $\left(U_{h}^{p}, \psi_{h}^{p}\right)$ in $\mathbb{X}_{h} \times \mathbb{M}_{h}^{\gamma_{0}}$ such that

$$
\begin{aligned}
& \forall V_{h} \in \mathbb{X}_{h}, \quad a\left(U_{h}^{p}, V_{h}\right)+b\left(V_{h}, \psi_{h}^{p}\right)=\mathcal{L}\left(V_{h}\right), \\
& \forall \chi_{h} \in \mathbb{M}_{h}^{\gamma_{0}}, \quad b\left(U_{h}^{p}, \chi_{h}\right)=\varepsilon_{p} c\left(\psi_{h}^{p}, \chi_{h}\right) .
\end{aligned}
$$

The main and well-known advantage of this problem, see [16, Chap. I, §4.3] for instance, is that it allows for uncoupling the two unknowns $U_{h}$ and $\psi_{h}$, and so solving only the equation of $U_{h}$, which is less expensive than problem (3.3). Indeed, let $\Pi_{h}$ denote the orthogonal projection operator from $H_{\gamma_{0}}^{1}(\omega)$ onto $\mathbb{M}_{h}^{\gamma_{0}}$ associated with the scalar product $c(\cdot, \cdot)$. Then, the second line of problem (4.2) can be written equivalently

$$
\psi_{h}^{p}=\frac{1}{\varepsilon_{p}} \Pi_{h}\left(r_{h}^{p} \cdot a_{3}\right)
$$

Inserting this last equation in the first line of (4.2) leads to the problem

Find $U_{h}^{p}$ in $\mathbb{X}_{h}$ such that

$$
\forall V_{h} \in \mathbb{X}_{h}, \quad a\left(U_{h}^{p}, V_{h}\right)+\frac{1}{\varepsilon_{p}} c\left(\Pi_{h}\left(\boldsymbol{r}_{h}^{p} \cdot \boldsymbol{a}_{3}\right), \Pi_{h}\left(\boldsymbol{s}_{h} \cdot \boldsymbol{a}_{3}\right)\right)=\mathcal{L}\left(V_{h}\right) .
$$

To prove the well-posedness of problem (4.2), we recall the following ellipticity property from [8, Lemma 3.3]: There exists a constant $c>0$ such that

$$
\forall V=(\boldsymbol{v}, \boldsymbol{s}) \in \mathbb{X}(\omega), \quad a(V, V)+c\left(\boldsymbol{s} \cdot \boldsymbol{a}_{3}, \boldsymbol{s} \cdot \boldsymbol{a}_{3}\right) \geq c\|V\|_{\mathbb{X}(\omega)}^{2} .
$$

To go further, we need a technical result which involves the Lagrange interpolation operator $\mathcal{I}_{h}$ at the vertices of all elements of $\mathcal{T}_{h}$ with values in $\mathbb{M}_{h}$ (note that this operator preserves the nullity on $\gamma_{0}$ ).

Lemma 4.1. Assume that the function $\boldsymbol{a}_{3}$ belongs to $H^{s}(\omega)^{3}$, for a real number $s>2$. The following estimate holds for any $s_{h}$ in $\left(\mathbb{M}_{h}^{\gamma_{0}}\right)^{3}$,

$$
\left|s_{h} \cdot a_{3}-\mathcal{I}_{h}\left(s_{h} \cdot a_{3}\right)\right|_{H^{1}(\omega)} \leq c h\left|s_{h}\right|_{H^{1}(\omega)^{3}} .
$$

Proof: We recall from [6, Lemme IX.1.2] for instance the estimate, for all $K$ in $\mathcal{T}_{h}$,

$$
\left|s_{h} \cdot a_{3}-\mathcal{I}_{h}\left(s_{h} \cdot a_{3}\right)\right|_{H^{1}(K)} \leq c h_{K}\left|s_{h} \cdot a_{3}\right|_{H^{2}(K)} .
$$


We observe that, since $s_{h}$ is affine on $K$,

$$
\left(\partial_{\alpha \beta}\left(s_{h} \cdot a_{3}\right)\right)_{\mid K}=\partial_{\alpha} s_{h} \cdot \partial_{\beta} a_{3}+\partial_{\beta} s_{h} \cdot \partial_{\alpha} a_{3}+s_{h} \cdot \partial_{\alpha \beta} a_{3} .
$$

Combining the two previous lines yields that, for any pair of positive real numbers $\left(q, q^{*}\right)$ such that $\frac{1}{q}+\frac{1}{q^{*}}=\frac{1}{2}$,

$\left|s_{h} \cdot a_{3}-\mathcal{I}_{h}\left(s_{h} \cdot a_{3}\right)\right|_{H^{1}(K)} \leq c h_{K}\left(\left\|s_{h}\right\|_{H^{1}(K)^{3}}\left\|a_{3}\right\|_{W^{1, \infty}(K)^{3}}+\left\|s_{h}\right\|_{L^{q^{*}}(K)^{3}}\left\|a_{3}\right\|_{W^{2, q}(K)^{3}}\right)$.

Summing the square of this inequality on the $K$ and using a Hölder inequality give

$$
\left|s_{h} \cdot a_{3}-\mathcal{I}_{h}\left(s_{h} \cdot a_{3}\right)\right|_{H^{1}(\omega)} \leq c h\left(\left\|s_{h}\right\|_{H^{1}(\omega)^{3}}\left\|a_{3}\right\|_{W^{1, \infty}(\omega)^{3}}+\left\|s_{h}\right\|_{L^{q^{*}}(\omega)^{3}}\left\|a_{3}\right\|_{W^{2, q}(\omega)^{3}}\right) .
$$

Using the imbedding of $H^{s}(\omega)$ into $W^{1, \infty}(\omega)$, choosing $q$ such that $H^{s}(\omega)$ is imbedded in $W^{2, q}(\omega)$ and using the imbedding of $H^{1}(\omega)$ into the corresponding $L^{q^{*}}(\omega)$ lead to the desired estimate.

By noting that

$$
\begin{aligned}
c\left(\Pi_{h}\left(s_{h} \cdot a_{3}\right), \Pi_{h}\left(s_{h} \cdot a_{3}\right)\right) & -c\left(s_{h} \cdot a_{3}, s_{h} \cdot a_{3}\right) \\
& =c\left(\left(\Pi_{h}-\mathrm{Id}\right)\left(s_{h} \cdot a_{3}-\mathcal{I}_{h}\left(s_{h} \cdot a_{3}\right)\right), s_{h} \cdot a_{3}\right)
\end{aligned}
$$

we derive from (4.5) and Lemma 4.1 that

$$
\forall V_{h}=\left(\boldsymbol{v}_{h}, \boldsymbol{s}_{h}\right) \in \mathbb{X}_{h}, \quad a\left(V_{h}, V_{h}\right)+c\left(\Pi_{h}\left(\boldsymbol{s}_{h} \cdot \boldsymbol{a}_{3}\right), \Pi_{h}\left(\boldsymbol{s}_{h} \cdot \boldsymbol{a}_{3}\right)\right) \geq\left(c-c^{\prime} h\right)\left\|V_{h}\right\|_{\mathbb{X}(\omega)}^{2},
$$

so that this form is elliptic for $h$ small enough. We also recall from [8, Thm 5.7] the inf-sup condition: There exists a constant $c>0$ independent of $h$ such that

$$
\forall \chi_{h} \in \mathbb{M}_{h}^{\gamma_{0}}, \quad \sup _{V_{h} \in \mathbb{X}_{h}} \frac{b\left(V_{h}, \chi_{h}\right)}{\left\|V_{h}\right\|_{\mathbb{X}(\omega)}} \geq c\left\|\chi_{h}\right\|_{H^{1}(\omega)} .
$$

Combining all this with [16, Chap. I, Thm 4.3] leads to the well-posedness result.

Proposition 4.2. Assume that the function $a_{3}$ belongs to $H^{s}(\omega)^{3}$, for a real number $s>2$. There exists $h_{*}>0$ such that, for all $h \leq h_{*}$ and for any data $(f, N, M)$ in $L^{2}(\omega)^{3} \times L^{2}\left(\gamma_{1}\right)^{3} \times L^{2}\left(\gamma_{1}\right)^{3}$, problem (4.2) admits a unique solution $\left(U_{h}^{p}, \psi_{h}^{p}\right)$ in $\mathbb{X}_{h} \times \mathbb{M}_{h}^{\gamma_{0}}$. Moreover, this solution satisfies

$$
\left\|U_{h}^{p}-U_{h}\right\|_{\mathbb{X}(\omega)}+\left\|\psi_{h}^{p}-\psi_{h}\right\|_{H^{1}(\omega)} \leq c \varepsilon_{p}\|\mathcal{L}\| .
$$

Remark 4.3. Writing a more sophisticated version of Lemma 4.1 yields that, when $s>1$, inequality (4.7) can be replaced by

$$
a\left(V_{h}, V_{h}\right)+c\left(\Pi_{h}\left(s_{h} \cdot a_{3}\right), \Pi_{h}\left(s_{h} \cdot a_{3}\right)\right) \geq\left(c-c^{\prime} h^{s-1}\right)\left\|V_{h}\right\|_{\mathbb{X}(\omega)}^{2} .
$$


Thus Proposition 4.2 still holds when $a_{3}$ belongs to $H^{s}(\omega)^{3}$, for a real number $s>1$, however for a lower value of $h_{*}$. So the previous result applies to piecewise regular shells with curvature discontinuities.

To perform the a posteriori analysis of problem (4.2), we introduce the next problem:

Find $\left(U^{p}, \psi^{p}\right)$ in $\mathbb{X}(\omega) \times \mathbb{M}(\omega)$ such that

$$
\begin{aligned}
& \forall V \in \mathbb{X}(\omega), \quad a\left(U^{p}, V\right)+b\left(V, \psi^{p}\right)=\mathcal{L}(V), \\
& \forall \chi \in \mathbb{M}(\omega), \quad b\left(U^{p}, \chi\right)=\varepsilon_{p} c\left(\psi^{p}, \chi\right),
\end{aligned}
$$

It follows from (4.5) and (2.19) that it admits a unique solution. Thus, following the approach of $[5, \S 3.3]$, we prove an upper bound for the error in two steps, relying on the triangle inequalities

$$
\begin{aligned}
\left\|U-U_{h}^{p}\right\|_{\mathbb{X}(\omega)} \leq\left\|U-U^{p}\right\|_{\mathbb{X}(\omega)}+\left\|U^{p}-U_{h}^{p}\right\|_{\mathbb{X}(\omega)}, \\
\quad\left\|\psi-\psi_{h}^{p}\right\|_{H^{1}(\omega)} \leq\left\|\psi-\psi^{p}\right\|_{H^{1}(\omega)}+\left\|\psi^{p}-\psi_{h}^{p}\right\|_{H^{1}(\omega)} .
\end{aligned}
$$

To estimate the penalization error, we observe that the following residual equations are satisfied

$$
\begin{aligned}
& \forall V \in \mathbb{X}(\omega), \quad a\left(U-U^{p}, V\right)+b\left(V, \psi-\psi^{p}\right)=0, \\
& \forall \chi \in \mathbb{M}(\omega), \quad b\left(U-U^{p}, \chi\right)=-\varepsilon_{p} c\left(\psi^{p}, \chi\right),
\end{aligned}
$$

So we are led to define the error indicator

$$
\eta^{p}=\varepsilon_{p}\left|\psi_{h}^{p}\right|_{H^{1}(\omega)}
$$

Theorem 4.4. There exists a constant $c$ independent of $p$ and $h$ such that the following a posteriori error estimate holds between the solutions $(U, \psi)$ of problem $(2.16)$ and $\left(U^{p}, \psi^{p}\right)$ of problem (4.10)

$$
\left\|U-U^{p}\right\|_{\mathbb{X}(\omega)}+\left\|\psi-\psi^{p}\right\|_{H^{1}(\omega)} \leq c\left(\eta^{p}+\varepsilon_{p}\left\|\psi^{p}-\psi_{h}^{p}\right\|_{H^{1}(\omega)}\right) .
$$

There exists a constant $c^{\prime}$ independent of $p$ and $h$ such that the following bound holds for the indicator $\eta^{p}$ defined in (4.13)

$$
\eta^{p} \leq c^{\prime}\left\|U-U^{p}\right\|_{\mathbb{X}(\omega)}+\varepsilon_{p}\left\|\psi^{p}-\psi_{h}^{p}\right\|_{H^{1}(\omega)} .
$$

Proof: Using (2.13) and (2.19), we easily derive from (4.12) (see [16, Chap. I, Cor. 4.1]) that

$$
\left\|U-U^{p}\right\|_{\mathbb{X}(\omega)}+\left\|\psi-\psi^{p}\right\|_{H^{1}(\omega)} \leq c \varepsilon_{p}\left|\psi^{p}\right|_{H^{1}(\omega)} .
$$

On the other hand, taking $\chi$ equal to $\psi^{p}$ in (4.12) yields

$$
\varepsilon_{p}\left|\psi^{p}\right|_{H^{1}(\omega)} \leq c\left\|U-U^{p}\right\|_{\mathbb{X}(\omega)} .
$$

Estimates (4.14) and (4.15) are then easily derived thanks to triangle inequalities. 
To estimate the discretization error, using once more the ideas in $[5, \S 3.3]$, we introduce the space

$$
\mathbb{Y}(\omega)=\mathbb{X}(\omega) \times \mathbb{M}(\omega),
$$

and the bilinear form defined on $\mathbb{Y}(\omega) \times \mathbb{Y}(\omega)$ by

$$
\mathcal{A}_{p}(U, \psi ; V, \chi)=a(U, V)+b(V, \psi)+b(U, \chi)-\varepsilon_{p} c(\psi, \chi) .
$$

Lemma 4.5. There exists a constant $\mu>0$ such that the following inf-sup condition holds

$$
\forall(U, \psi) \in \mathbb{Y}(\omega), \quad \sup _{(V, \chi) \in \mathbb{Y}(\omega)} \frac{\mathcal{A}_{p}(U, \psi ; V, \chi)}{\|V\|_{\mathbb{X}(\omega))}+\|\chi\|_{H^{1}(\omega)}} \geq \mu\left(\|U\|_{\mathbb{X}(\omega)}+\|\psi\|_{H^{1}(\omega)}\right) .
$$

Proof: For any $(\mathcal{F}, \mathcal{G})$ in the dual of $\mathbb{Y}(\omega)$, it can be checked from (4.5) and (2.19) that the problem: Find $(W, \xi)$ in $\mathbb{Y}(\omega)$ such that

$$
\begin{array}{ll}
\forall V \in \mathbb{X}(\omega), & a(W, V)+b(V, \xi)=\mathcal{F}(V), \\
\forall \chi \in \mathbb{M}(\omega), \quad b(W, \chi)-\varepsilon_{p} c(\xi, \chi)=\mathcal{G}(\chi),
\end{array}
$$

has a unique solution. Moreover, using (2.19) in the first line of (4.19) yields

$$
c_{\sharp}\|\xi\|_{H^{1}(\Omega)} \leq c\|W\|_{\mathbb{X}(\omega)}+\|\mathcal{F}\|_{\mathbb{X}(\omega)^{\prime}} \cdot
$$

We now proceed in two steps.

1) Owing to the inf-sup condition (2.19), there exists a $W_{0}$ in $\mathbb{X}(\omega)$ such that

$$
\forall \chi \in \mathbb{M}(\omega), \quad b\left(W_{0}, \chi\right)=\varepsilon_{p} c(\xi, \chi)+\mathcal{G}(\chi)
$$

and

$$
c_{\sharp}\left\|W_{0}\right\|_{\mathbb{X}(\omega)} \leq \varepsilon_{p}|\xi|_{H^{1}(\omega)}+\|\mathcal{G}\|_{H_{\gamma_{0}}^{1}(\omega)^{\prime}} .
$$

The function $W-W_{0}$ now belongs to $\mathbb{V}(\omega)$ and satisfies

$$
\forall V \in \mathbb{V}(\omega), \quad a\left(W-W_{0}, V\right)=\mathcal{F}(V)-a\left(W_{0}, V\right) .
$$

Using the ellipticity property $(2.13)$ of $a(\cdot, \cdot)$ on $\mathbb{V}(\omega)$ thus yields

$$
\left\|W-W_{0}\right\|_{\mathbb{X}(\omega)} \leq c\left(\|\mathcal{F}\|_{\mathbb{X}(\omega)^{\prime}}+\left\|W_{0}\right\|_{\mathbb{X}(\omega)}\right) .
$$

Combining this estimate with a triangle inequality, (4.21) and next (4.20) gives

$$
\|W\|_{\mathbb{X}(\omega)} \leq c\left(\|\mathcal{F}\|_{\mathbb{X}(\omega)^{\prime}}+\|\mathcal{G}\|_{H_{\gamma_{0}}^{1}(\omega)^{\prime}}\right)+c^{*} \varepsilon_{p}\|W\|_{\mathbb{X}(\omega)} .
$$

Setting $\varepsilon^{*}=\min \left\{1, \frac{1}{2 c^{*}}\right\}$ (note that $\varepsilon^{*}$ only depends on $c_{\sharp}$ and the norm of $a(\cdot, \cdot)$ ), we derive that, for all $\varepsilon_{p} \leq \varepsilon^{*}$,

$$
\|W\|_{\mathbb{X}(\omega)} \leq c\left(\|\mathcal{F}\|_{\mathbb{X}(\omega)^{\prime}}+\|\mathcal{G}\|_{H_{\gamma_{0}}^{1}(\omega)^{\prime}}\right) .
$$


2) On the other hand, denoting by $\mathcal{R}$ the Riesz isomorphism from $H_{\gamma_{0}}^{1}(\omega)^{\prime}$ onto $H_{\gamma_{0}}^{1}(\omega)$ and setting $W=(\boldsymbol{w}, \boldsymbol{t})$, we observe that the second line in (4.19) can equivalently be written as

$$
\xi=\frac{1}{\varepsilon_{p}}\left(t \cdot a_{3}-\mathcal{R G}\right) .
$$

Combining this equation with the first line in (4.19) leads to

$$
\forall V \in \mathbb{X}(\omega), \quad a(W, V)+\frac{1}{\varepsilon_{p}} c\left(\boldsymbol{t} \cdot \boldsymbol{a}_{3}, s \cdot \boldsymbol{a}_{3}\right)=\mathcal{F}(V)+\frac{1}{\varepsilon_{p}} c\left(\mathcal{R} \mathcal{G}, s \cdot \boldsymbol{a}_{3}\right) .
$$

In the case $\varepsilon^{*} \leq \varepsilon_{p} \leq 1$ (so that $1 \leq \frac{1}{\varepsilon_{p}} \leq \frac{1}{\varepsilon^{*}}$ ), estimate (4.22) also follows from the ellipticity property (4.5) and the positivity of $c\left(\boldsymbol{s} \cdot \boldsymbol{a}_{3}, \boldsymbol{s} \cdot \boldsymbol{a}_{3}\right)$.

In both cases $\varepsilon_{p} \leq \varepsilon^{*}$ and $\varepsilon^{*}<\varepsilon_{p} \leq 1$, inserting (4.22) into (4.20) yields that

$$
\|\xi\|_{H^{1}(\Omega)} \leq c\left(\|\mathcal{F}\|_{\mathbb{X}(\omega)^{\prime}}+\|\mathcal{G}\|_{H_{\gamma_{0}}^{1}(\omega)^{\prime}}\right) .
$$

It thus follows from (4.22) and (4.23) that the operator: $(\mathcal{F}, \mathcal{G}) \mapsto(W, \xi)$ is an isomorphism from $\mathbb{Y}(\omega)^{\prime}$ onto $\mathbb{Y}(\omega)$. So standard arguments [16, Chap. I, Lemma 4.1] give the inf-sup condition (4.18).

The residual equation for problem (4.2) can be written as

$$
\begin{aligned}
\forall(V, \chi) \in \mathbb{Y}(\omega), \forall\left(V_{h}, \chi_{h}\right) \in \mathbb{X}_{h} \times \mathbb{M}_{h}^{\gamma_{0}}, & \\
\mathcal{A}_{p}\left(U^{p}-U_{h}^{p}, \psi^{p}-\psi_{h}^{p} ; V, \chi\right)=\mathcal{L}\left(V-V_{h}\right) & -a\left(U_{h}^{p}, V-V_{h}\right)-b\left(V-V_{h}, \psi_{h}^{p}\right) \\
& -b\left(U_{h}^{p}, \chi-\chi_{h}\right)+\varepsilon_{p} c\left(\psi_{h}^{p}, \chi-\chi_{h}\right) .
\end{aligned}
$$

So it is very similar to (3.6), except for the last term in the right-hand side.

We now define the error indicators: For each $K$ in $\mathcal{T}_{h}$,

$$
\tilde{\eta}_{K}=\tilde{\eta}_{K 1}+\tilde{\eta}_{K 2}+\tilde{\eta}_{K 3}
$$

where $\tilde{\eta}_{K 1}$ and $\tilde{\eta}_{K 2}$ are defined as $\eta_{K 1}$ and $\eta_{K 2}$ by (3.20) and (3.21) but with $\left(U_{h}, \psi_{h}\right)$ replaced by $\left(U_{h}^{p}, \psi_{h}^{p}\right)$ while $\tilde{\eta}_{K 3}$ involves a further term:

$$
\begin{aligned}
\tilde{\eta}_{K 3}= & h_{K}\left\|\partial_{\alpha}\left(\partial_{\alpha} r_{h}^{p} \cdot \boldsymbol{a}_{3 h}+\boldsymbol{r}_{h}^{p} \cdot \boldsymbol{d}_{\alpha}^{h}-\varepsilon_{p} \partial_{\alpha} \psi_{h}^{p}\right)\right\|_{L^{2}(K)} \\
& +\sum_{e \in \mathcal{E}_{K} \backslash \mathcal{E}_{K}^{1}} h_{e}^{\frac{1}{2}}\left\|\left[\partial_{\nu}\left(\boldsymbol{r}_{h} \cdot \boldsymbol{a}_{3}^{h}-\varepsilon_{p} \psi_{h}^{p}\right)\right]_{e}\right\|_{L^{2}(e)}+\sum_{e \in \mathcal{E}_{K}^{1}} h_{e}^{\frac{1}{2}}\left\|\partial_{\nu}\left(\boldsymbol{r}_{h} \cdot \boldsymbol{a}_{3}^{h}-\varepsilon_{p} \psi_{h}^{p}\right)\right\|_{L^{2}(e)} .
\end{aligned}
$$

We do not give the proof of the next theorem. Indeed, thanks to the inf-sup condition (4.18), it reduces to the evaluation of the right-hand side of (4.24), which is performed exactly as for Theorem 3.6.

Theorem 4.6. For any data $(f, N, M)$ in $L^{2}(\omega)^{3} \times L^{2}\left(\gamma_{1}\right)^{3} \times L^{2}\left(\gamma_{1}\right)^{3}$, the following a posteriori error estimate holds between the solution $\left(U^{p}, \psi^{p}\right)$ of problem (4.10) and the solution $\left(U_{h}^{p}, \psi_{h}^{p}\right)$ of problem (4.2)

$$
\left\|U^{p}-U_{h}^{p}\right\|_{\mathbb{X}(\omega)}+\left\|\psi^{p}-\psi_{h}^{p}\right\|_{H^{1}(\omega)} \leq c\left(\left(\sum_{K \in \mathcal{T}_{h}}\left(\tilde{\eta}_{K}^{2}+\varepsilon_{K}^{(d) 2}\right)\right)^{\frac{1}{2}}+\varepsilon_{h}^{(c)}\right) .
$$


Similarly, bounding the $\tilde{\eta}_{K}$ relies on appropriate choices of the pair $(V, \chi)$ in $(4.24)$ (with $\left(V_{h}, \chi_{h}\right)$ equal to zero), combined with usual inverse inequalities, see [22, §1.2].

Theorem 4.7. For any data $(\boldsymbol{f}, \boldsymbol{N}, \boldsymbol{M})$ in $L^{2}(\omega)^{3} \times L^{2}\left(\gamma_{1}\right)^{3} \times L^{2}\left(\gamma_{1}\right)^{3}$, the following bound holds for each indicator $\tilde{\eta}_{K}$ defined in (4.25),

$$
\left.\tilde{\eta}_{K} \leq c\left(\left\|U^{p}-U_{h}^{p}\right\|_{\mathbb{X}\left(\omega_{K}\right)}+\left\|\psi^{p}-\psi_{h}^{p}\right\|_{H^{1}\left(\omega_{K}\right)}+\left(\sum_{\kappa \subset \omega_{K}} \varepsilon_{\kappa}^{(d) 2}\right)\right)^{\frac{1}{2}}+\varepsilon_{h}^{(c)}\right) .
$$

When combining Theorems 4.4, 4.6 and 4.7, we observe that, up to the terms $\varepsilon_{K}^{(d)}$ and $\varepsilon_{h}^{(c)}$, the full error

$$
E_{p h}=\left\|U-U^{p}\right\|_{\mathbb{X}(\omega)}+\left\|U^{p}-U_{h}^{p}\right\|_{\mathbb{X}(\omega)}+\left\|\psi-\psi^{p}\right\|_{H^{1}(\omega)}+\left\|\psi^{p}-\psi_{h}^{p}\right\|_{H^{1}(\omega)},
$$

is equivalent to the quantity

$$
\eta_{p h}=\eta^{p}+\left(\sum_{K \in \mathcal{T}_{h}} \tilde{\eta}_{K}^{2}\right)^{\frac{1}{2}}
$$

with equivalence constants independent of $\varepsilon_{p}$ and $h$. So these estimates are fully optimal. Moreover it appears in the next section that, even though the two parts of the error are not completely uncoupled in (4.14) and (4.15), the $\tilde{\eta}_{K}$ are a very efficient tool to adapt the mesh independently of the value of $\varepsilon_{p}$ (analogous properties can be found in [5, Figs. $1 \& 2$ ] for a different problem). 


\section{The adaptivity strategy and numerical experiments.}

We now describe how the error indicators exhibited in Sections 3 and 4 can be used to adapt the mesh for each discrete problem. Next, we apply these strategies to some numerical experiments.

\subsection{Adaptivity strategy for the first discrete problem}

This strategy is very simple and consists of two steps. We fix a tolerance $\eta^{*}>0$.

MESh INITIALIZATION STEP. We choose a triangulation $\mathcal{T}_{h}^{0}$ such that the quantity

$$
\left(\sum_{K \in \mathcal{T}_{h}} \varepsilon_{K}^{(d) 2}\right)^{\frac{1}{2}}+\varepsilon_{h}^{(c)}
$$

is smaller than $\eta^{*}$ (we recall that the $\varepsilon_{K}^{(d)}$ and $\varepsilon_{h}^{(c)}$ are defined in (3.17) and (3.18), respectively). This induces a refinement of the mesh where the data $(f, N, M)$ are singular and also near the curvature discontinuities, which seems very natural.

Mesh adAPtation step. Assume that the triangulation $\mathcal{T}_{h}^{n}$ is known. We compute the discrete solution of problem (3.3) corresponding to this triangulation, and the error indicators $\eta_{K}$ defined in (3.19) to (3.22). Let also $\bar{\eta}_{h}$ denote the mean value of the $\eta_{K}$. The triangulation $\mathcal{T}_{h}^{n}$ is then refined and coarsened according to the next criterion: The diameter of a new element contained in $K$ or containing $K$ is proportional to $h_{K}$ times the ratio $\bar{\eta}^{h} / \eta_{K}$. This gives rise to the new triangulation $\mathcal{T}_{h}^{n+1}$. We refer to [15, Chap. 21] for the processus leading to the construction of such a triangulation.

The mesh adaptation step is then iterated either until the quantity $\left(\sum_{K \in \mathcal{T}_{h}^{n}} \eta_{K}^{2}\right)^{\frac{1}{2}}$ becomes smaller than $\eta^{*}$ (when possible) or a finite number of times.

\subsection{Adaptivity strategy for the second discrete problem}

A strategy aimed to simultaneously optimize the penalty parameter and adapt the mesh is proposed in $[5, \S 5]$. However, in the present situation, first computations show that the main part of the solution, i.e., $\left(\boldsymbol{u}_{h}, \boldsymbol{r}_{h}-\left(\boldsymbol{r}_{h} \cdot \boldsymbol{a}_{3}\right) \boldsymbol{a}_{3}\right)$, is nearly independent of the choice of $\varepsilon_{p}$, so that applying this strategy seems impossible. Thus, we now describe a simpler algorithm. We fix two tolerances $\eta^{*}$ and $\eta^{\diamond}$, with $\eta^{\diamond}$ much larger than $\eta^{*}$ (typically, $\eta^{*}$ is equal to $10^{-7}$ and $\eta^{\diamond}$ is equal to $10^{-2}$ ).

Mesh initialization SteP. It is the same as in Section 5.1.

Penalty initialization SteP. An initial value $\varepsilon^{0}$ is also chosen. Then, we make a first computation for this value of $\varepsilon^{0}$ and the triangulation $\mathcal{T}_{h}^{0}$, and also compute $\eta^{p}$ and the $\eta_{K}$. If

$$
\eta^{p} \leq \eta^{\diamond}\left(\sum_{K \in \mathcal{T}_{h}^{0}} \eta_{K}^{2}\right)^{\frac{1}{2}}
$$


we take $\varepsilon_{p}$ equal to $\varepsilon^{0}$ and go to the next step. Otherwise, we divide $\varepsilon^{0}$ by the ratio $\eta^{p} / \eta^{\diamond}\left(\sum_{K \in \mathcal{T}_{h}^{0}} \eta_{K}^{2}\right)^{\frac{1}{2}}$ and make a new computation.

Mesh AdAptation STEP. It is exactly the same as in Section 5.1 and is iterated until the quantity $\left(\sum_{K \in \mathcal{T}_{h}^{n}} \eta_{K}^{2}\right)^{\frac{1}{2}}$ becomes smaller than $\eta^{*}$ (when possible) or a finite number of times.

\subsection{Numerical experiments}

The numerical experiments that we now present have been performed on the finite element code FreeFem++, see [17]. In order to check the efficiency of our adaptivity strategy, we have decided to present numerical experiments for the same geometries and data as in $[8, \S 6.2 \& 6.3]$.

As standard for problems in elasticity and more specifically for shell models (see [1, $\S 3.1]$ for instance), both problems (3.3) and (4.2) result into very ill-conditioned linear systems. This is due to the physical parameters of the model and, among them, to the small thickness of the shell. For these reasons, we have decided to use the direct solver UMFPACK relying on the $L U$ factorizatrion of the global sparse matrix, see [17] for more details.

We first consider a hyperbolic paraboloid shell. The reference domain $\omega$ is the square

$$
\omega=\{(x, y) ;|x|+|y| \leq b \sqrt{2}\}
$$

as illustrated in $[1, \S 1.3 .3 \& 2.4 .2]$, and the chart $\varphi$ is defined by

$$
\varphi(x, y)=\left(x, y, \frac{c}{2 b^{2}}\left(x^{2}-y^{2}\right)\right)^{T}
$$

We choose here

$$
b=50 \mathrm{~cm}, \quad c=10 \mathrm{~cm} \text {. }
$$

The thickness of the shell is $e=0.8 \mathrm{~cm}$. We assume that the shell is clamped on the whole boundary, i.e. $\gamma_{0}=\partial \omega$, and that it is subjected to a uniform pressure. The mechanical data are

$$
E=2.8 \times 10^{9} \mathrm{~Pa}, \quad \nu=0.4 .
$$

Note that the symmetry properties of the domain and the data allow us to solve the discrete problem only on the triangle $\omega^{\prime}$ with vertices $(0,0)^{T},(b \sqrt{2}, 0)^{T}$ and $(0, b \sqrt{2})^{T}$. We refer to $[8, \S 6.2]$ for the artificial conditions issued from the symmetry conditions.

Figure 1 presents the initial mesh. We then take $\varepsilon_{p}$ equal to $10^{-1}$. Figure 2 presents the final adapted meshes according to the strategy described in Sections 5.1 and 5.2, without penalization (left part) and with penalization (right part). Figure 3 presents the "over-deformed" shell, namely the surface $\varphi(x)+1000 u(x), x \in \bar{\omega}$, where the solution is computed with penalization, seen from the top and bottom sides. 


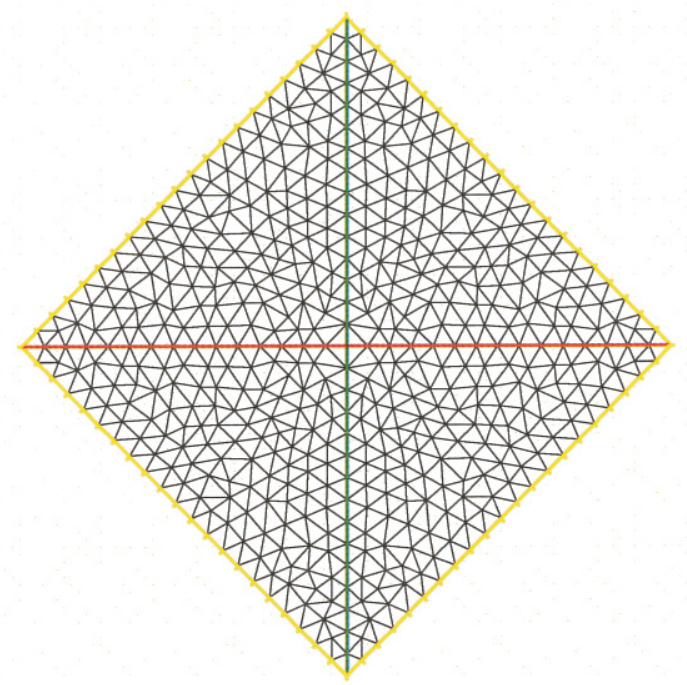

Figure 1: The initial mesh
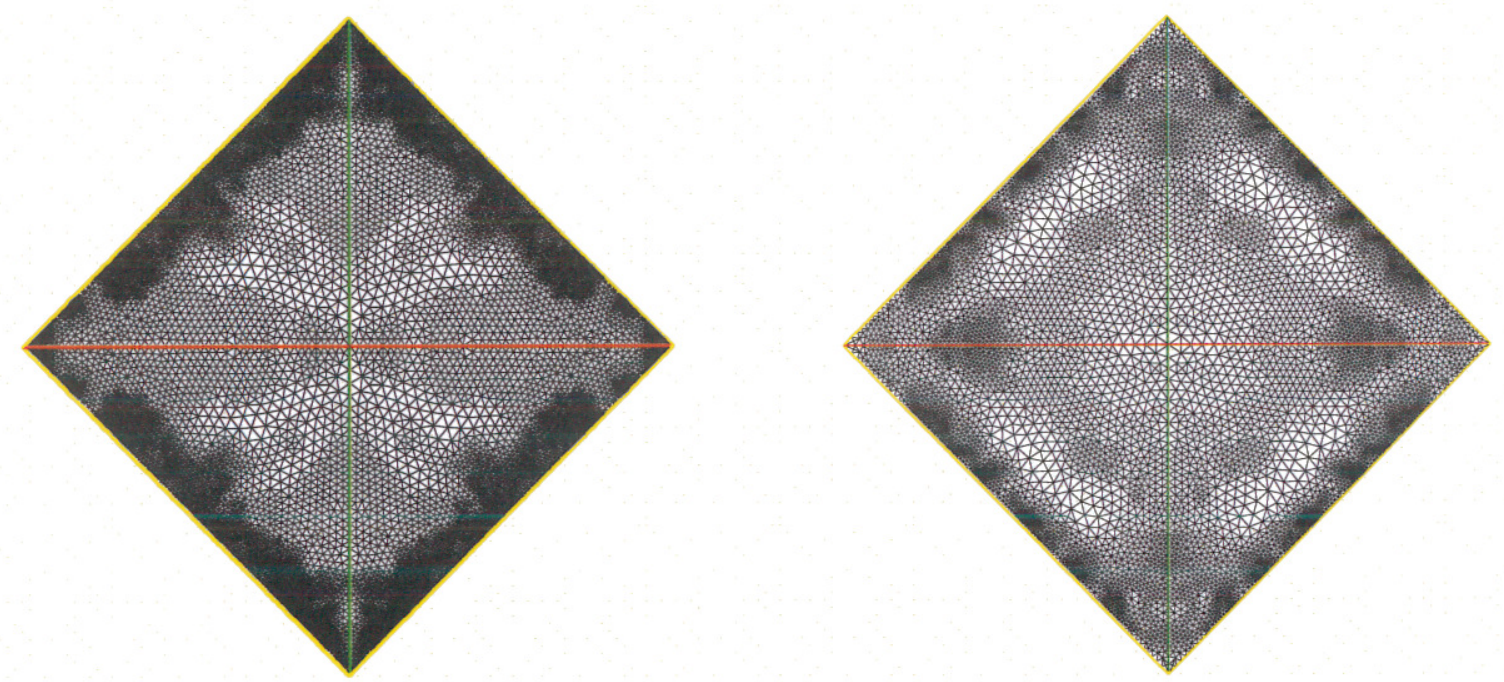

Figure 2: The adapted meshes without and with penalization
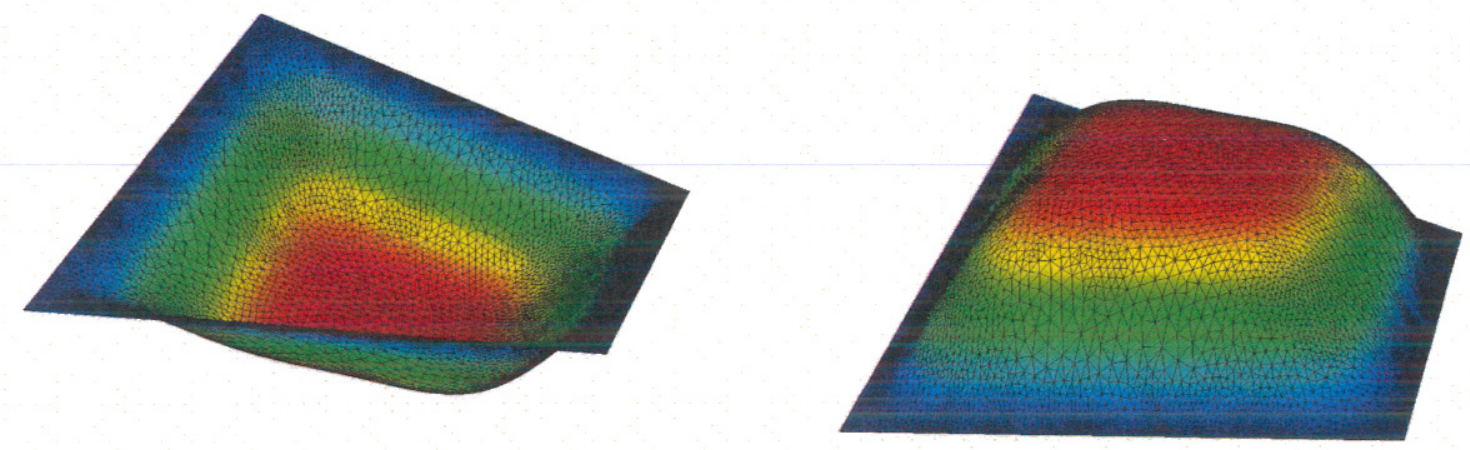

Figure 3: The "over-deformed" shell 
Next, we consider a plane-cylinder $W^{2, \infty}$-shell: The shell consists of a plane part and a cylindrical part with a $\mathscr{C}^{1}$-join; see Figure 4 . The reference domain $\omega$ of the midsurface is given by

$$
\omega=]-R, R[\times]-L, L[,
$$

and the chart $\varphi$ is defined by

$$
\varphi(x, y)= \begin{cases}(x, y, 0)^{T} & \text { if } x<0 \\ (R \sin (x / R), y, R(1-\cos (x / R)))^{T} & \text { if } x \geq 0 .\end{cases}
$$

We take here

$$
R=15 \mathrm{~m}, \quad L=7.5 \mathrm{~m} \text {. }
$$

The thickness of the shell is $e=7.5 \mathrm{~cm}$. In Figure 4, "transported triangulation" means $\varphi\left(\mathcal{T}_{h}\right)$.

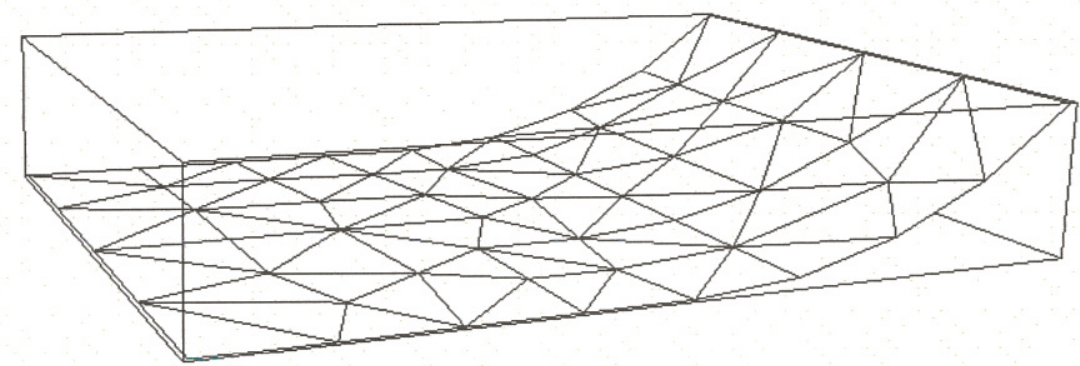

Figure 4: The geometry of the shell and its initial transported triangulation

The mechanical data are

$$
E=2.1 \times 10^{10} \mathrm{~Pa}, \quad \nu=0 .
$$

There also, the shell is submitted to a uniform downward pressure. Concerning boundary conditions, we consider the case of hard clamping on two edges

$$
\left.\gamma_{0}=\{-R\} \times\right]-L, L[\cup\{R\} \times]-L, L[
$$

and the shell is free on its remaining edges.

Figure 5 presents the final meshes

- without penalization on the left part, where "transported triangulation" here means $\left(\varphi+10 u_{h}\right)\left(\mathcal{T}_{h}\right)$

- with penalization on the right part, for $\varepsilon_{p}=10^{-3}$ and where "transported triangulation" means $\left(\varphi+10 \boldsymbol{u}_{h}^{p}\right)\left(\mathcal{T}_{h}\right)$.

Figure 6 presents the isovalue curves of the first and third Cartesian components $u_{h 1}^{p}$ and $u_{h 3}^{p}$ of the displacement obtained by the discretization with penalization $\left(\varepsilon_{p}=10^{-3}\right)$; note however that its analogue obtained without penalization is exactly the same. 

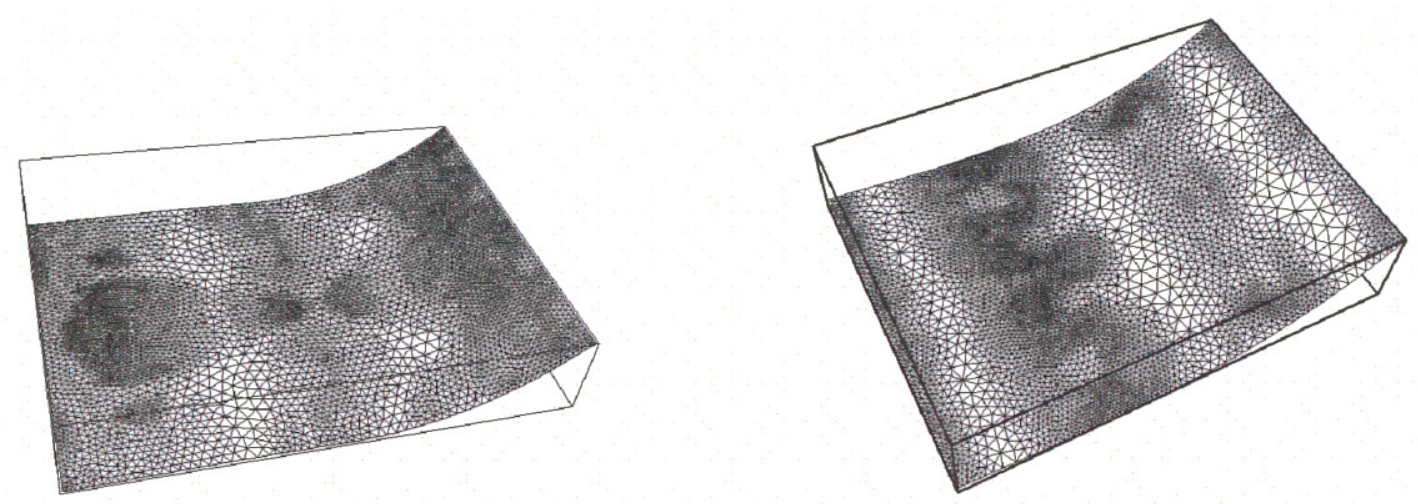

Figure 5: The adapted transported triangulations without and with penalization
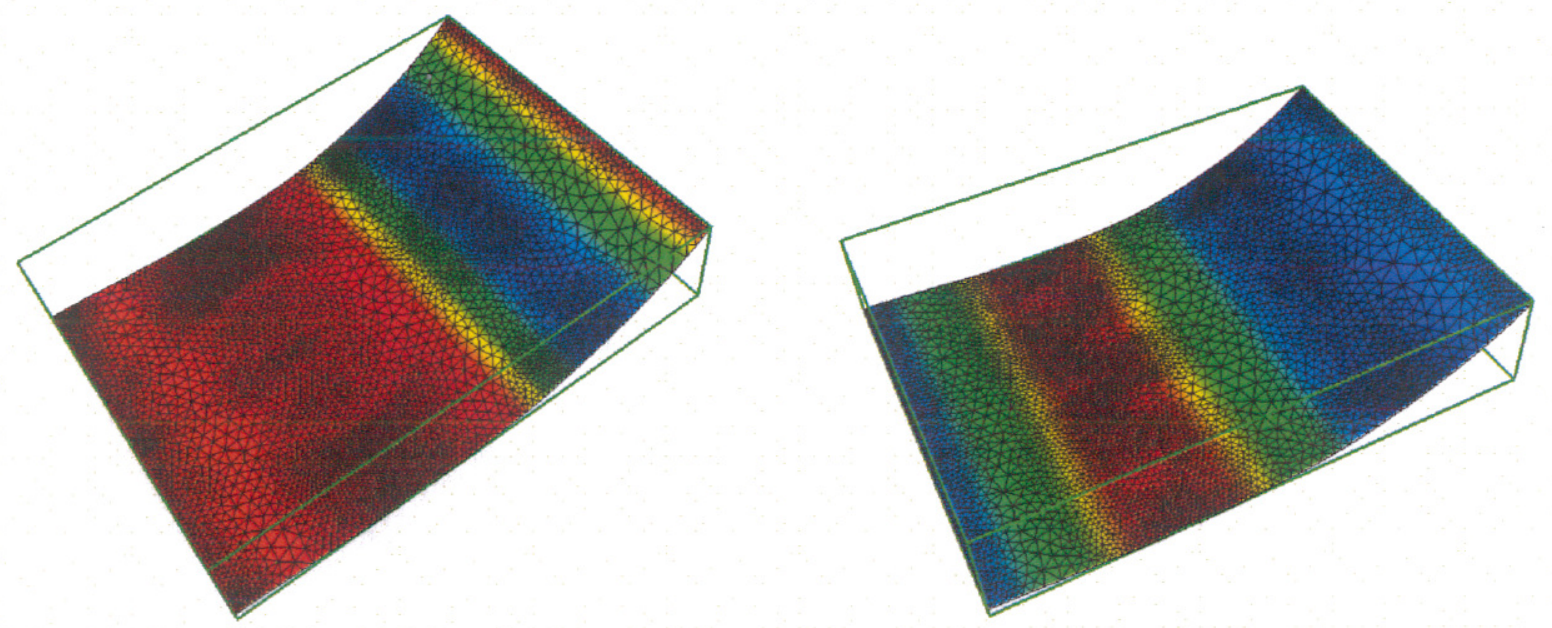

Figure 6: The Cartesian components $u_{h 1}^{p}$ and $u_{h 3}^{p}$ of the displacement

We have not presented the component $u_{h 2}^{p}$ of the dislacement since it is nearly zero. In fact, both horizontal displacements are very small $\left(\leq 10^{-5} \mathrm{~cm}\right)$ in comparison with the vertical one (of order $1.5 \mathrm{~cm}$ ); this is in good agreement with the physics of the problem since the force applied to the shell is vertical. 


\section{References}

[1] M. Bernadou - Méthodes d'éléments finis pour les problèmes de coques minces, Collection "Recherches en Mathématiques Appliquées" 33, Masson (1994).

[2] M. Bernadou, P.G. Ciarlet - Sur l'ellipticité du modèle linéaire de coques de W.T. Koiter, in Computing Methods in Applied Sciences and Engineering, R. Glowinski and J.-L. Lions eds., Lecture Notes in Economics and Mathematical Systems, vol. 134, Springer, Berlin (1976), 89-136.

[3] C. Bernardi, A. Blouza - Spectral discretization of a Naghdi shell model, SIAM J. Numer. Anal. 45 (2007), 2653-2670.

[4] C. Bernardi, T. Chacón Rebollo, E. Chacón Vera, D. Franco Coronil - A non-overlapping domain decomposition method motivated by a posteriori error analysis, Appl. Num. Math. (2008), in press.

[5] C. Bernardi, V. Girault, F. Hecht - A posteriori analysis of a penalty method and application to the Stokes problem, Math. Models and Methods in Applied Sciences 13 (2003), 1599-1628.

[6] C. Bernardi, Y. Maday, F. Rapetti - Discrétisations variationnelles de problèmes aux limites elliptiques, Collection "Mathématiques et Applications" 45, Springer-Verlag (2004).

[7] A. Blouza - Existence et unicité pour le modèle de Nagdhi pour une coque peu régulière, C. R. Acad. Sci. Paris, Série I 324 (1997), 839-844.

[8] A. Blouza, F. Hecht, H. Le Dret - Two finite element approximations of Naghdi's shell model in Cartesian coordinates, SIAM J. Numer. Anal. 44 (2006), 636-654.

[9] A. Blouza, H. Le Dret - Existence and uniqueness for the linear Koiter model for shells with little regularity, Quarterly of Applied Mathematics LVII (1999), 317-337.

[10] A. Blouza, H. Le Dret - Nagdhi's shell model: Existence, uniqueness and continuous dependence on the midsurface, Journal of Elasticity 64 (2001), 199-216.

[11] M. Carrive, P. Le Tallec, J. Mousso - Approximation par éléments finis d'un modèle de coques géométriquement exact, Revue Européenne des Éléments Finis 4 (1995), 633-662.

[12] C. Carstensen, J. Schöberl - Residual-based a posteriori error estimate for a mixed ReißnerMindlin plate finite element method, Numer. Math. 103 (2006), 225-250.

[13] D. Chapelle, A. Ferent, K.J. Bathe - 3D-shell elements and their underlying mathematical model, Math. Models and Methods in Applied Sciences 14 (2004), 105-142.

[14] P.G. Ciarlet - Mathematical Elasticity, Volume III: Theory of Shells, North-Holland (2000).

[15] P.J. Frey, P.-L. George - Maillages, applications aux éléments finis, Hermès (1999).

[16] V. Girault, P.-A. Raviart - Finite Element Methods for Navier-Stokes Equations, Theory and Algorithms, Springer-Verlag (1986).

[17] F. Hecht, O. Pironneau - FreeFem++, see www.freefem.org.

[18] N. Kerdid, P. Mato Eiroa - Conforming finite element approximation for shells with little regularity, Comput. Methods in Applied Mech. and Engrg. 188 (2000), 95-107. 
[19] C. Lacour, Y. Maday — La méthode des éléments avec joint appliquée aux méthodes d'approximations "discrete Kirchhoff triangles", C. R. Acad. Sci. Paris, Série I 326 (1998), 1237-1242.

[20] P. Le Tallec, J. Mandel, M. Vidrascu - A Neumann-Neumann domain decomposition algorithm for solving plate and shell problems, SIAM J. Numer. Anal. 35 (1998), 836-867.

[21] P. Le Tallec, S. Mani - Analyse numérique d'un modèle de coque de Koiter discrétisé en base cartésienne par éléments finis DKT, Modél. Math. Anal. Numér. 32 (1998), 433-450.

[22] R. Verfürth - A Review of A Posteriori Error Estimation and Adaptive Mesh-Refinement Techniques, Wiley \& Teubner (1996). 